COMMUNICATIONS IN

ANALYSIS AND GEOMETRY

Volume 14, Number 1, 25-58, 2006

\title{
From Sparks to Grundles - Differential Characters
}

\author{
Reese Harvey and Blaine LaWson
}

\begin{abstract}
We introduce a new homological machine for the study of secondary geometric invariants. The objects, called spark complexes, occur in many areas of mathematics. The theory is applied here to establish the equivalence of a large family of spark complexes which appear naturally in geometry, topology and physics. These complexes are quite different. Some of them are purely analytic, some are simplicial, some are of Čech-type, and many are mixtures. However, the associated theories of secondary invariants are all shown to be canonically isomorphic. Numerous applications and examples are explored.
\end{abstract}

\section{Introduction.}

The point of this paper is to present a general homological apparatus for the study of sparks, differential characters, gerbes and analogous objects. This overarching theory encompasses all the known theories equivalent to differential characters, as well as many new ones, and it establishes this equivalence. The basic algebra is elementary and self-contained. Nevertheless, it enables one to rigorously establish canonical isomorphisms between the quite disparate approaches to the theory: the original Cheeger-Simons formulation, the de Rham-Federer theory using currents, the Cech-de Rham formulation connected with gerbes, and many others. It also provides a homological framework for the development of new secondary theories which will be pursued in separate papers.

In the first section, we introduce the concept of a homological spark complex and its associated group of homological spark classes. (These play roles analogous to cochain complexes and their associated groups of cohomology classes in standard homological algebra.) We establish a basic $3 \times 3$ grid of short exact sequences with the group of homological spark classes in the center. The spark complexes of interest in this paper will be referred to collectively as $(\mathbf{R}, \mathbf{Z})$-spark complexes. For these complexes, the grid captures all of the basic short exact sequences.

We then introduce the notion of compatible spark complexes and show that compatible complexes have naturally isomorphic groups of spark 
classes. This is the tool that enables us to rigorously unify the many approaches to the $(\mathbf{R}, \mathbf{Z})$ theory.

The next part of the paper is devoted to studying specific examples. We shall present: holonomy maps, differential characters, de Rham-Federer spark classes, hyperspark classes, smooth hyperspark classes, grundles $(n$ gerbes with connection), cochain hyperspark classes, Cheeger-Simons spark classes, current cochain spark classes, and prove that all of these concepts are equivalent. The diagram

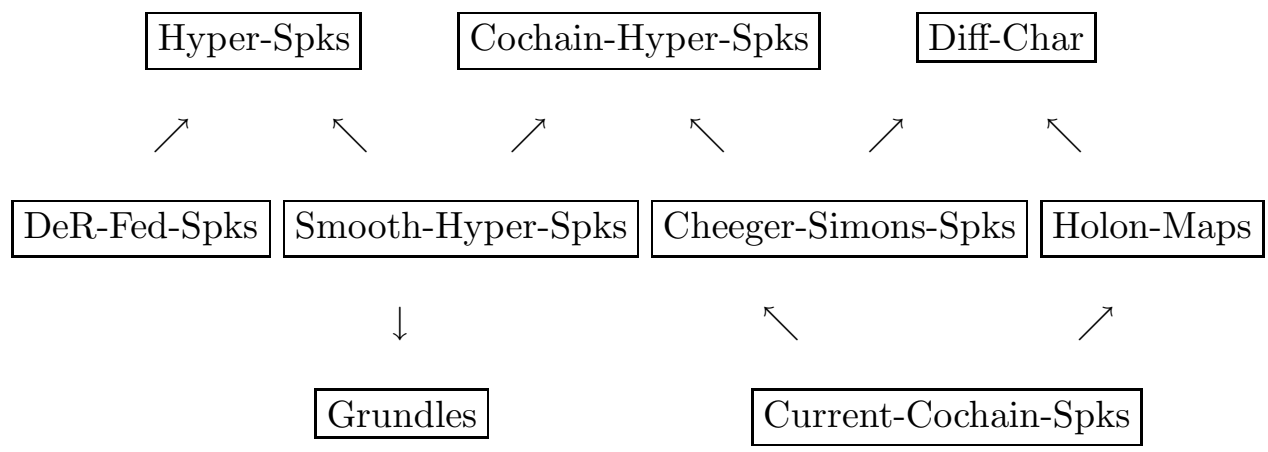

\section{$(\mathbf{R}, \mathbf{Z})$-Spark Complexes}

provides a convenient summary. The arrows in this diagram represent the direction of the inclusion maps at the level of spark complexes. This part of the paper is organized as follows.

In Section 2, we recall the de Rham-Federer spark complexes which first appeared in [8] and [10] and were systematically studied in [17]. There are in fact many such complexes, from the most general one involving all currents, to quite restricted ones in which only forms with $L_{\text {loc }}^{1}$-coefficients appear as sparks [17, Section 2].

In Section 3, we define the complex of hypersparks whose compatibility with all the de Rham-Federer spark complexes establishes the isomorphisms between their groups of spark classes.

In Section 4, we introduce smooth hypersparks and show that they form a compatible subcomplex of the hypersparks.

In Section 5, we present the concept of grundles of degree $k$ and the notion of gauge equivalence. Grundles of degree 1 are just Cech presentations of hermitian line bundles with unitary connection, and "gauge equivalence" corresponds to the standard equivalence as unitary bundles with connection. 
Grundles of degree 2 similarly correspond to (abelian) gerbes with connection, and "gauge equivalence" corresponds to the standard equivalence found in the literature $[20,1]$.

Grundles of degree $k$ and smooth hypersparks of degree $k$ are essentially the same. They differ only in the lowest order component where one is obtained from the other by choosing logarithms (or inversely by exponentiation). In particular, we show that gauge equivalence classes of grundles are isomorphic to the corresponding classes of smooth hypersparks (which are isomorphic to groups of spark classes of all the aforementioned spark complexes).

In Section 6, we consider Cheeger-Simons sparks which are the sparks most closely related to differential characters as originally defined in [3]. These sparks are shown to be compatible with the smooth hypersparks via a larger complex which encompasses them both (cochain hypersparks).

In Section 7, we examine Cheeger-Simons theory. Recall that in [3], a differential character of degree $k$ is defined to be a homomorphism

$$
h: Z_{k}(X) \longrightarrow \mathbf{R} / \mathbf{Z} \quad \text { such that } \quad d h \equiv \phi \quad \bmod \mathbf{Z}
$$

for some smooth $(k+1)$-form $\phi$ on $X$. Here, $Z_{k}(X)=\left\{c \in C_{k}(X): \partial c=\right.$ $0\}$ where $C_{k}(X)$ is the group of $C^{\infty}$ singular $k$-chains in $X$ with integer coefficients, and the congruence above means that $h(\partial c)-\int_{c} \phi \in \mathbf{Z}$ for all $c \in C_{k+1}(X)$. We show that the group $\operatorname{DiffChar}^{k}(X)$ is isomorphic to Cheeger-Simons spark classes, and hence to each group of spark classes appearing in Sections 2-6.

From a geometric point of view perhaps the most natural starting point for the $(\mathbf{R}, \mathbf{Z})$-theory is to define "holonomy maps". Quite surprisingly, this concept does not seem to be part of the literature. Note that every $k$-chain $c \in C_{k}(X)$ defines a current, that is, a linear functional $\widetilde{c}$ on the space $\mathcal{E}^{k}(X)$ of smooth $k$-forms on $X$, by setting $\widetilde{c}(\psi)=\int_{c} \psi$. Let $\widetilde{Z}_{k}(X)$ be the group of currents coming from cycles in $Z_{k}(X)$ in this way. (These are current chains in the sense of de Rham.) There is a short exact sequence

$$
0 \rightarrow N_{k}(X) \rightarrow Z_{k}(X) \stackrel{\rho}{\longrightarrow} \widetilde{Z}_{k}(X) \rightarrow 0
$$

where $\rho(z)=\widetilde{z}$ and $N_{k}(X)$ is the group of null cycles on $X$, the cycles over which all forms integrate to be 0 . Note for example that a compact oriented submanifold $M \subset X$ of dimension $k$ defines an element $[M] \in$ $\widetilde{Z}_{k}(X)$. Among the many cycles $z$ with $\rho(z)=[M]$ are those arising from smooth triangulations of $M$. 
In Section 8, we define the set of degree $k$ holonomy maps to be

$$
\begin{aligned}
\operatorname{Hol}(X) \equiv\left\{H \in \operatorname{Hom}\left(\widetilde{Z}_{k}(X), \mathbf{R} / \mathbf{Z}\right): d H\right. & \equiv \phi \bmod \mathbf{Z} \\
& \text { for some } \left.\phi \in \mathcal{E}^{k+1}(X)\right\}
\end{aligned}
$$

In Theorem 8.2, we show that every differential character $h$ as above can be written in the form $h=H \circ \rho$ for a homomorphism $H: \widetilde{Z}_{k}(X) \rightarrow \mathbf{R} / \mathbf{Z}$. In other words, there is an isomorphism

$$
\operatorname{DiffChar}^{k}(X) \cong \operatorname{Hol}(X) .
$$

It is well known that in degree 1 , differential characters are isomorphic to gauge equivalence classes of principal $S^{1}$-bundles with connection, and that the isomorphism is given by the classical holonomy of the connection. The above results generalize this statement to grundles of all degrees. In particular, the analogue of (0.1) for grundles (abelian gerbes with connection) recaptures results in [22].

This analogue implies that the holonomy of any grundle over a compact oriented submanifold $M$ is independent of any choice of triangulation of $M$. However, given a triangulation it is interesting to find combinatorial formulae for the holonomy in terms of the grundle data. A general formula of this type is derived in Section 9. In degree 1, this formula computes the holonomy around a loop by integration of the connection 1-forms on subarcs where the bundle is trivialized and the change of trivialization at the endpoints of the arcs. The corresponding formula (9.4) for the holonomy of a gerbe with connection involves integration of the gerbe data over the 0-,1and 2 -faces.

There are many $(\mathbf{R}, \mathbf{Z})$ homological spark complexes which were not mentioned in Sections 2-6. We briefly discuss two useful ones in Section 10.

We note that the discussion here was restricted to the case of "integer coefficients". However, one can easily replace $\mathbf{Z}$ with any proper subring $\Lambda \subset \mathbf{R}$. For example, in the discussion of Section 3, one replaces $C^{*}(\mathcal{U}, \mathbf{Z}) \subset$ $C^{*}(\mathcal{U}, \mathbf{R})$ with $C^{*}(\mathcal{U}, \Lambda) \subset C^{*}(\mathcal{U}, \mathbf{R})$, and everything goes through.

Historically, the spark equation and spark equivalence first appeared in [8] and [10] and were systematically developed in [17], where PoincaréPontrjagin duality for differential characters was established. The concept of smooth hypersparks can be found in the work of Freed-Witten [7], and Picken [21], and the concept of grundles (sometimes called $n$-gerbes with connection) can be found in many places, e.g. [20]. Cheeger-Simons cochain sparks appear explicitly in the papers of Cheeger and Simons $[2,3]$ as "liftings" of differential characters $\phi: Z_{k}(X) \rightarrow \mathbf{R} / \mathbf{Z}$ to homomorphisms 
$\widetilde{\phi}: Z_{k}(X) \rightarrow \mathbf{R}$. However, they do not systematically study the spark equation and spark equivalence.

In Section 11, we discuss many applications and examples. Topics include: ring structure, functoriality, Gysin maps and Thom homomorphisms, secondary characteristic classes, flat sparks, projective bundles, Hodge sparks, Morse sparks, the Wess-Zumino term, refined integer SteifelWhitney classes, and linear dependency sparks. In each case, the main concern is to determine which points of view represented in the diagram above are most illuminating.

In this paper, our attention is focused on $(\mathbf{R}, \mathbf{Z})$-spark complexes. However, there exist many spark complexes whose associated class groups are quite different and interesting.

For example on complex manifolds, one can define a $\bar{\partial}$-analogue of differential characters by applying (a slight generalization of) the homological machinery developed here to spark complexes involving forms and currents of type $(0, q)$. There are several compatible formulations of the theory which yield the same $\bar{\partial}$-spark classes. One formulation uses a smooth ČechDolbeault complex which is related (as in this paper) to $\bar{\partial}$-grundles. In low degrees, these grundles have beautiful geometric interpretations as in the classical case. However, there is quite a different Dolbeault-Federer approach to $\bar{\partial}$-spark classes which is purely analytic. It enables us to establish functoriality and to define a $*$-product which makes these groups into a graded ring. The equivalence of these spark complexes is proved by a larger hyperspark complex compatible with them both, as in this paper. One interesting by-product of the discussion is an acyclic resolution of the sheaf $\mathcal{O}^{*}$ by forms and currents. All these results will appear in [15].

We emphasize that there exist many further interesting spark complexes. Some involve $(p, q)$-forms and are related to Deligne cohomology (see [15]). Others arise in a quite general way from interesting double complexes. Among these are complexes related to the arithmetic Chow groups of Gillet and Soulé [16].

Note. In discussing certain spark complexes, we talk freely about sheaves of germs of currents of various types. For a unified approach to the cohomology of current complexes (such as flat, integrally flat, integral, etc.), the reader is referred to the Appendix A in [18].

For simplicity, all complexes in this paper will be complexes of abelian groups, i.e., Z-modules. 


\section{Homological Sparks.}

A spark complex consists of a differential complex $F^{*}, d$, and two subcomplexes $E^{*} \subset F^{*}$ and $I^{*} \subset F^{*}$ which satisfy the following properties:

(A) $H^{k}\left(E^{*}\right) \cong H^{k}\left(F^{*}\right), k=0,1, \ldots$

(B) $E^{k} \cap I^{k}=\{0\}, k=1,2, \ldots$

It follows that

$$
E^{0} \cap I^{0}=H^{0}\left(I^{*}\right) \subset H^{0}\left(E^{*}\right),
$$

since $a \in E^{0} \cap I^{0}$ implies $d a \in E^{1} \cap I^{1}=\{0\}$ and $H^{0}\left(I^{*}\right) \subset H^{0}\left(F^{*}\right)=$ $H^{0}\left(E^{*}\right)$.

Definition 1.1. An element $a \in F^{k}$ with the property that

$$
d a=\phi-r \quad(\text { the Spark equation) }
$$

where $\phi \in E^{k+1}$ and $r \in I^{k+1}$ is a homological spark of degree $k$. Two homological sparks are equivalent if their difference is of the form

$$
d b+s
$$

with $b \in F^{k-1}$ and $s \in I^{k}$. Given a homological spark $a \in F^{k}$, let $\hat{a}$ denote the equivalence class containing the spark $a$, and let $\widehat{\mathbf{H}}^{k}$ denote the group of homological spark classes.

Sometimes, it is convenient to set $\widehat{\mathbf{H}}^{-1}=H^{0}\left(I^{*}\right)$. (The spark equation is $0=\phi-r$.)

Lemma 1.2. Each homological spark $a \in F^{k}$ uniquely determines $\phi \in E^{k+1}$ and $r \in I^{k+1}$, and $d \phi=0, d r=0$. Moreover, $\phi \in E^{k+1}$ and $[r] \in H^{k+1}\left(I^{*}\right)$ only depend on the spark class $\hat{a} \in \widehat{\mathbf{H}}^{k}$.

Consequently, we may define

$$
\delta_{1}: \widehat{\mathbf{H}}^{k} \rightarrow E^{k+1} \quad \text { and } \quad \delta_{2}: \widehat{\mathbf{H}}^{k} \rightarrow H^{k+1}\left(I^{*}\right)
$$

by $\delta_{1} \hat{a}=\phi$, and $\delta_{2} \hat{a}=[r]$. Given a spark class $\alpha \in \widehat{\mathbf{H}}^{k}$, the element $\phi=\delta_{1} \alpha \in E^{k+1}$ will be referred to as the curvature of $\alpha$, and the class $\delta_{2} \alpha \in H^{k+1}\left(I^{*}\right)$ will be referred to as the divisor class of $\alpha$.

Proof. Uniqueness of $\phi$ and $r$ is immediate from Axiom (B). Since $d \phi=d r$, Axiom (B) implies both must vanish. Changing the homological spark $a$ 
by adding $d b$ does not change $\phi$ or $r$. Changing the homological spark $a$ by adding $s \in I^{k}$ does not change $\phi$ and changes $r$ to $r-d s$ (Again using (B)).

There is an obvious notion of morphism of spark complexes.

Let $Z_{I}^{k}\left(E^{*}\right)$ denote the space of cycles $\phi \in E^{k}$ which are $F^{*}$-homologous to some $r \in I^{k}$, i.e. the space of possible curvature elements. Let us also define

$$
\begin{aligned}
H_{I}^{k}\left(F^{*}\right) \equiv \text { Image }\left\{H^{k}\left(I^{*}\right)\right. & \left.\rightarrow H^{k}\left(F^{*}\right)\right\}=\operatorname{Ker}\left\{H^{k}\left(F^{*}\right) \rightarrow H^{k}\left(F^{*} / I^{*}\right)\right\} \\
H^{k+1}\left(F^{*}, I^{*}\right) \equiv & \operatorname{Ker}\left\{H^{k+1}\left(I^{*}\right) \rightarrow H^{k+1}\left(F^{*}\right)\right\} \\
& =\operatorname{Image}\left\{H^{k}\left(F^{*} / I^{*}\right) \rightarrow H^{k+1}\left(I^{*}\right)\right\} .
\end{aligned}
$$

Finally, let $\widehat{\mathbf{H}}_{E}^{k}$ denote the space of spark classes that can be represented by a homological spark $a \in E^{k}$. Note that

$$
\widehat{\mathbf{H}}_{E}^{k} \cong E^{k} / Z_{I}^{k}
$$

Proposition 1.3. The following diagram commutes, and each row and column is exact $(k>0)$ :

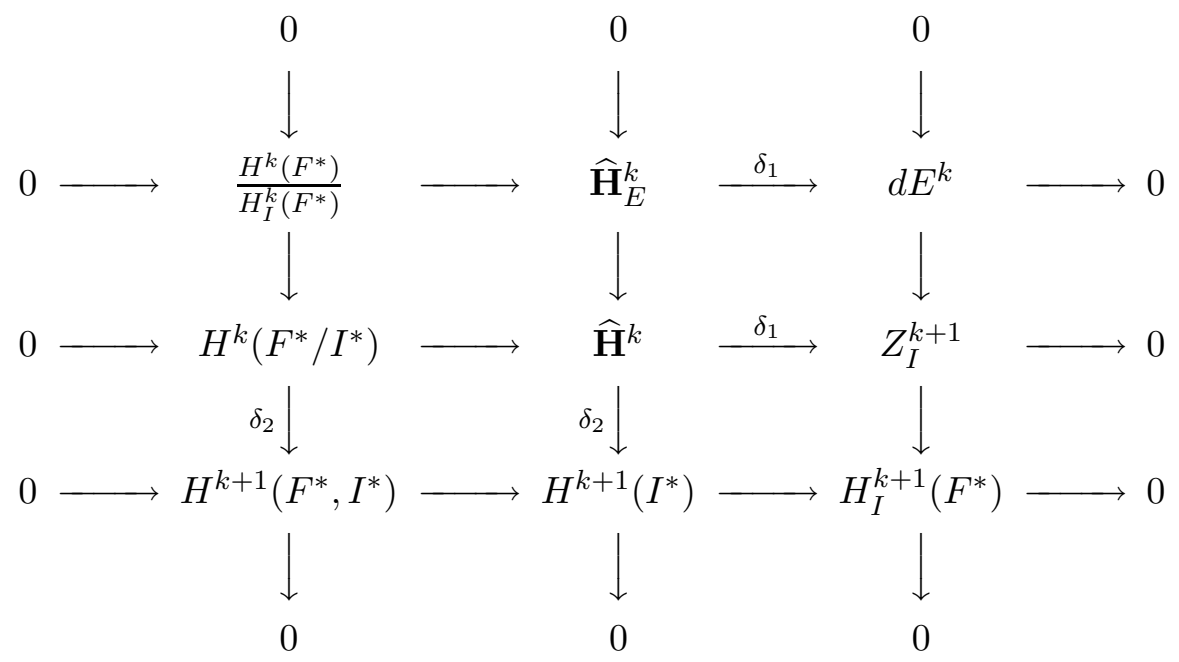

The proof is straightforward, see [17] or Remark 1.6 below.

A spark complex

$$
\begin{array}{r}
E^{*} \subset F^{*} \\
\cup \\
I^{*}
\end{array}
$$


is said to be a subspark complex of another spark complex

$$
\begin{array}{r}
\bar{E}^{*} \subset \bar{F}^{*} \\
\cup \\
\bar{I}^{*}
\end{array}
$$

if $F^{*} \subset \bar{F}^{*}$ is a differential subcomplex with $E^{*}=\bar{E}^{*}$ the same, and $I^{*} \subset \bar{I}^{*}$ such that:

$$
H^{*}\left(I^{*}\right) \cong H^{*}\left(\bar{I}^{*}\right) \text {. }
$$

Two spark complexes are said to be compatible if they can be embedded as subspark complexes of a spark complex.

Theorem 1.4. Given two compatible spark complexes, there is a natural isomorphism $\widehat{\mathbf{H}}^{k} \cong \widehat{\overline{\mathbf{H}}}^{k}, k=-1,0,1, \ldots$.

The following Lemma will be used twice in the proof of this Proposition.

Lemma 1.5. Suppose $F^{*}, d$ is a subdifferential complex of $\bar{F}^{*}, d$. Then, the assertion:

$$
H^{k}\left(F^{*}\right) \cong H^{k}\left(\bar{F}^{*}\right) \quad k=0,1, \ldots
$$

can be restated as follows: Given $g \in F^{p+1}$ and a solution $\alpha \in \bar{F}^{p}$ to the equation $d \alpha=g$ there exists $\gamma \in \bar{F}^{p-1}$, with

$$
f=\alpha+d \gamma \in F^{p} .
$$

(Not only is there a solution $f \in F^{p}$ to $d f=g$, but one which is homologous to the given solution $\alpha$ in $\bar{F}^{p}$.)

Proof. Since $H^{p+1}\left(F^{*}\right) \rightarrow H^{p+1}\left(\bar{F}^{*}\right)$ is injective, there exists a solution $h \in F^{p}$ with $d h=g$. Thus, $\alpha-h$ is $d$-closed. Since $H^{p}\left(F^{*}\right) \rightarrow H^{p}\left(\bar{F}^{*}\right)$ is surjective, there exist $\gamma \in \bar{F}^{p-1}$ such that $\alpha-h+d \gamma \in F^{p}$.

Proof of Theorem. We may assume that a subspark complex is given. Suppose $\bar{a} \in \bar{F}^{k}$ is an $(\bar{F}, \bar{I})$-spark, i.e. $d \bar{a}=\phi-\bar{r}$ with $\phi \in E^{k+1}$ and $\bar{r} \in \bar{I}^{k+1}$. Since $d r=0$ and $H^{k+1}\left(F^{*}\right) \cong H^{k+1}\left(\bar{F}^{*}\right)$ there exist $\bar{s} \in \bar{I}^{k}$, $r \in I^{k+1}$ with $\bar{r}=r-d \bar{s}$. Therefore, $d(\bar{a}-\bar{s})=\phi-r$. Since $\phi-r \in F^{k+1}$ and $H^{*}\left(F^{*}\right) \cong H^{*}\left(\bar{F}^{*}\right)$, the Lemma implies that there exist $\bar{b} \in \bar{F}$ with 
$a=\bar{a}-\bar{s}-d \bar{b} \in F^{k}$. This proves that the $(\bar{F}, \bar{I})$-spark $\bar{a} \in F^{k}$ is equivalent to an $(F, I)$-spark $a \in F^{k}$.

Suppose that $a \in F$ is an $(F, I)$-spark which is equivalent to zero as an $(\bar{F}, \bar{I})$-spark i.e. $d a=\phi-r$, with $\phi \in E^{k+1}, r \in I^{k+1}$ and $a=d \bar{b}+\bar{s}$ with $\bar{b} \in \bar{F}^{k-1}$ and $\bar{s} \in \bar{I}$. Then $d \bar{s}=\phi-r$ which implies that both $\phi=0$ and $d \bar{s}=-r$. Applying the Lemma to $I^{*} \subset \bar{I}^{*}$ there exist $\bar{t} \in \bar{I}^{k-1}, s \in I^{k}$ with $s=\bar{s}+d \bar{t}$. Therefore, $a-s=d(\bar{b}-\bar{t})$. Since $H^{k}\left(F^{*}\right) \cong H^{k}\left(\bar{F}^{*}\right)$, there exist $b \in F^{k-1}$ with $a-s=d b$. That is, $a$ is $(F, I)$ equivalent to zero.

Remark 1.6. We include a proof of the exactness of the $3 \times 3$ grid (Proposition 1.3) for the sake of completeness. The standard long exact sequence in homology associated with the short exact sequence of complexes, $0 \rightarrow I^{*} \rightarrow F^{*} \rightarrow F^{*} / I^{*} \rightarrow 0$, yields the equalities in (1.1) and (1.2) and the exactness of the left column and the bottom row. In the middle column, surjectivity of $\delta_{2}$ is equivalent to each cycle $r \in I^{k+1}$ satisfying a spark equation $d a=\phi-r$, which is easily verified. To see that $\delta_{2}$ has kernel $\widehat{\mathbf{H}}_{E}^{k}$, first note that if $\delta_{2} \alpha=0$, then we may choose a spark $a \in \alpha$ with $d a=\phi \in E^{k+1}$. The basic assumption (A) may be restated as follows. (This is just Lemma 1.5 applied to $E^{*} \subset F^{*}$.)

$(\mathrm{A})^{\prime}$ Given $a \in F^{k}$ with $\phi=d a \in E^{k+1}$, there exists $b \in F^{k-1}$ with $a+d b \in E^{k}$.

Hence, we can choose $a^{\prime}=a+d b \in \alpha$ with $a^{\prime} \in E^{k}$. This proves exactness of the middle column.

By the definition of $Z_{I}^{k+1}$, as the space of possible curvature elements, $\delta_{1}$ in the middle row is surjective. By various definitions the kernel of $\delta_{1}$ is $H^{k}\left(F^{*} / I^{*}\right)$.

Surjectivity in the right column asserts that the image of $Z_{I}^{k+1}$ equals Image $\left\{H^{k+1}\left(I^{*}\right) \rightarrow H^{k+1}\left(F^{*}\right)\right\}$. This means that the image of the set of all divisors classes equals the set of all curvature classes in $H^{k+1}\left(F^{*}\right)$, which is straightforward. The kernel is $d E^{k}$ since $F$-homology implies $E$-homology by assumption $(\mathrm{A})$.

It remains to show that:

$$
\left(\operatorname{ker} \delta_{1}\right) \cap\left(\operatorname{ker} \delta_{2}\right)=H^{k}\left(F^{*}\right) / H_{I}^{k}\left(F^{*}\right) .
$$

First, note that if $\delta_{1} \alpha=0$ and $\delta_{2} \alpha=0$, then there exists a spark $a \in \alpha$ with $d a=0$. Two such sparks are equivalent if $a-a^{\prime}=d b+s$ with $b \in F^{k-1}$, $s \in I^{k}$. Since $d a=d a^{\prime}=0$, it follows that $d s=0$ completing the proof of exactness in the $3 \times 3$ grid. Commutativity of the diagram is straightforward to verify. 
Remark 1.7. Proposition 1.4 can be strengthened by weakening the notion of compatible spark complexes to include the case of a chain map from $F^{*} \rightarrow \bar{F}^{*}$. See Section 10 for an application.

The remainder of this paper will be devoted to the study of a particular equivalence class of spark complexes, which will be collectively called $(\mathbf{R} / \mathbf{Z})$ spark complexes. The $3 \times 3$ grid for these complexes is given at the end of the next section.

\section{Sparks (de Rham-Federer).}

For completeness, we review in this section some of the results of [17].

Definition 2.1. A spark of degree $k$ is a current $\alpha \in \mathcal{D}^{\prime k}(X)$ with the property that

$$
d a=\phi-R, \quad(\text { The Spark equation) }
$$

where $\phi \in \mathcal{E}^{k+1}(X)$ is smooth and $R \in I \mathcal{F}^{k+1}(X)$ is integrally flat. Let $\mathcal{S}^{k}(X)$ denote the space of sparks of degree $k$.

Recall that a current $R$ is integrally flat if it can be written as $R=T+d S$ where $T$ and $S$ are locally rectifiable currents. The de Rham-Federer spark complex is obtained by taking $E^{k}=\mathcal{E}^{k}(X), F^{k}=\mathcal{D}^{\prime k}(X)$ and $I^{k}=I \mathcal{F}^{k}(X)$. Condition (A) is, of course, standard [5]. A proof of condition (B), i.e. that

$$
\mathcal{E}^{k}(X) \cap I \mathcal{F}^{k}(X)=\{0\} \quad k=1,2, \ldots
$$

is given in [17]. (See the proof of Lemma 1.3 on page 796.)

For convenience, the notion of equivalence is restated specifically for the de Rham-Federer complex.

Definition 2.2 (Spark Equivalence). Two sparks $a$ and $a^{\prime}$ are (spark) equivalent if there exists $b \in \mathcal{D}^{\prime k-1}(X)$, an arbitrary current, and $\mathcal{S} \in$ $I \mathcal{F}^{k}(X)$ an integrally flat current, with

$$
a-a^{\prime}=d b+S
$$

The equivalence class determined by a spark $a \in S^{k}(X)$ will be denoted $\hat{a}$, and the space of spark classes will be denoted by $\widehat{\mathbf{H}}_{\text {spark }}^{k}(X)$. 
There are many useful subspark complexes of the de Rham-Federer spark complex. For example, let $L_{\text {loc }}^{1}(X)^{k}$ denote the space of currents of degree $k$ which can be expressed as differential forms with locally Lebesgue integrable coefficients, and let $\widetilde{C}_{\mathrm{deR}}^{k}(X)$ denote the space of currents (the current chains of de Rham) that can locally be expressed as integration over a smooth singular $(n-k)$-chain with integer coefficients. Take $F^{k}=L_{\text {loc }}^{1}(X)^{k}+$ $d L_{\text {loc }}^{1}(X)^{k-1}$. Then, $I^{k}=\widetilde{C}_{\mathrm{deR}}^{k}(X) \subset F^{k}$ and one obtains

$$
\begin{gathered}
\widehat{\mathbf{H}}_{\mathrm{spark}}^{k}(X) \cong\left\{a \in L_{\mathrm{loc}}^{1}(X)^{k}: d a=\phi-R \text { with } \phi \in \mathcal{E}^{k+1}(X)\right. \text { and } \\
\left.R \in \widetilde{C}_{\mathrm{deR}}^{k+1}(X) \text { a current chain }\right\} /\left(d L_{\mathrm{loc}}^{1}(X)^{k}+\widetilde{C}_{\mathrm{deR}}^{k}(X)\right)
\end{gathered}
$$

All such modifications follow from Proposition 1.4.

Since $H^{k}\left(\mathcal{E}^{*}(X)\right)=H^{k}(X, \mathbf{R}), \quad H^{k}\left(I \mathcal{F}^{*}(X)\right)=H^{k}(X, \mathbf{Z})$, and $H^{k}\left(\mathcal{D}^{\prime *}(X) / I \mathcal{F}^{*}(X)\right)=H^{k}\left(X, S^{1}\right)$ (see [17] and [18]), the diagram in Proposition 1.3 can be rewritten as:

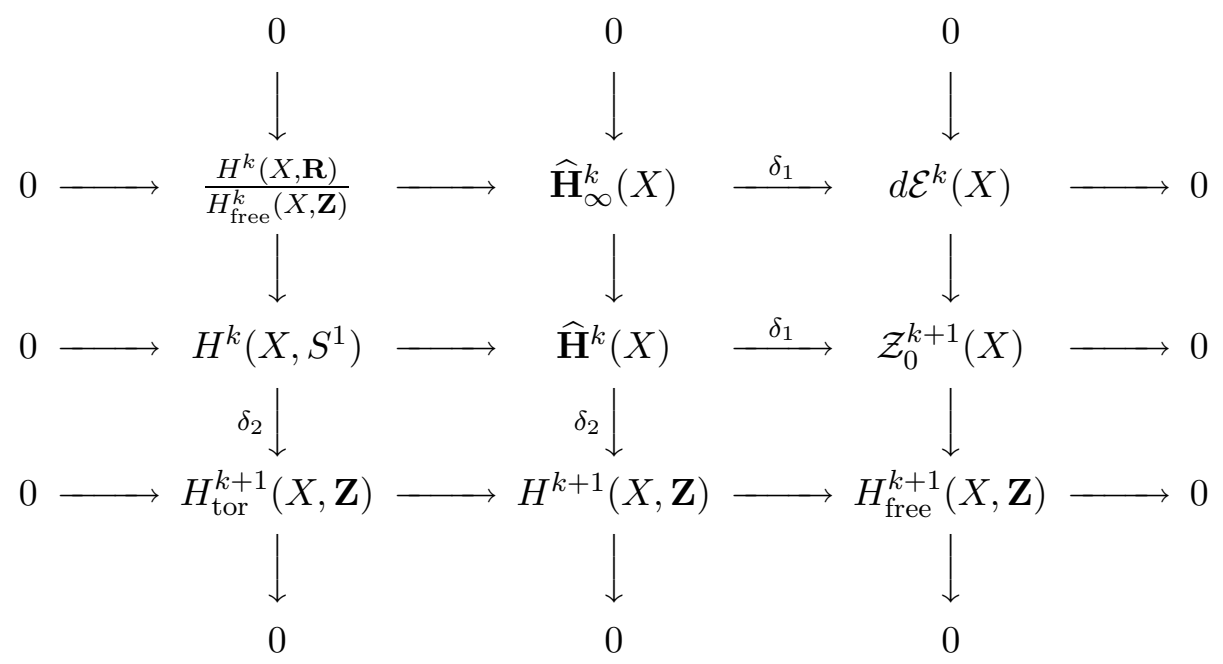

\section{Hypersparks.}

Suppose $\mathcal{U}=\left\{U_{i}\right\}$ is an open covering of $X$ (with each intersection $U_{I}$ contractible). Consider the Cech-Current bicomplex

$$
\bigoplus_{p, q \geq 0} C^{p}\left(\mathcal{U}, \mathcal{D}^{\prime q}\right)
$$

with total differential $D=(-1)^{q} \delta+d$. 
Note that $D^{2}=0$, and that both the horizontal and vertical homology is zero except along the two edges.

The kernel of $\delta$ on the left (vertical) edge $(p=0)$ is the de Rham complex $\mathcal{D}^{*}(X), d$ since

$$
0 \longrightarrow \mathcal{D}^{\prime q}(X) \longrightarrow C^{0}\left(\mathcal{U}, \mathcal{D}^{\prime q}\right) \stackrel{\delta}{\longrightarrow} C^{1}\left(\mathcal{U}, \mathcal{D}^{\prime q}\right)
$$

is exact.

The kernel of $d$ on the bottom edge $(q=0)$ is the Cech complex $C^{p}(\mathcal{U}, \mathbf{R})$, $\delta$ since

$$
0 \longrightarrow C^{p}(\mathcal{U}, \mathbf{R}) \longrightarrow C^{p}\left(\mathcal{U}, \mathcal{D}^{\prime o}\right) \stackrel{d}{\longrightarrow} C^{p}\left(\mathcal{U}, \mathcal{D}^{\prime 1}\right)
$$

is exact.

We will consider $C^{p}(\mathcal{U}, \mathbf{Z})$ as a subcomplex of $C^{p}(\mathcal{U}, \mathbf{R})$, and $C^{p}\left(\mathcal{U}, I F^{q}\right)$ as a subbicomplex of $C^{p}\left(\mathcal{U}, \mathcal{D}^{\prime q}\right)$.

Definition 3.1. A hyperspark of degree $k$ is an element

$$
A \in \bigoplus_{p+q=k} C^{p}\left(\mathcal{U}, \mathcal{D}^{\prime q}\right)
$$

with the property that

$$
D A=\phi-R \quad \text { (Hyperspark equation) }
$$

where $\phi \in \mathcal{E}^{k+1}(X)$ is smooth of bidegree $0, k+1$ and $R \in$ $\bigoplus_{p+q=k+1} C^{p}\left(\mathcal{U}, I F^{q}\right)$ is an integrally flat cochain.

Said differently, we are defining a spark complex by letting $\bar{F}^{*}=$ $\bigoplus_{p+q=*} C^{p}\left(\mathcal{U}, \mathcal{D}^{\prime q}\right)$ be the Čech-current bi-complex, and setting $\bar{E}^{*}=$ $\mathcal{E}^{*}(X) \subset C^{0}\left(\mathcal{U}, \mathcal{D}^{\prime q}\right)$ and $\bar{I}^{*}=\bigoplus_{p+q=*} C^{p}\left(\mathcal{U}, I F^{q}\right)$. Axioms A and B follow easily, and the notion of equivalence becomes:

Definition 3.2 (Equivalence). Two hypersparks $A$ and $\bar{A}$ are said to be equivalent if there exists $B \in \bigoplus_{p+q=k-1} C^{p}\left(\mathcal{U}, \mathcal{D}^{\prime q}\right)$ and $S \in$ $\bigoplus_{p+q=k} C^{p}\left(\mathcal{U}, I F^{q}\right)$ satisfying

$$
A-A^{\prime}=D B+S .
$$

The equivalence class determined by a hyperspark $A$ will be denoted by $\widehat{A}$, and the space of hyperspark classes will be denoted by $\widehat{\mathbf{H}}_{\text {hyperspark }}^{k}(X)$.

There is obviously a well defined map

$$
\widehat{\mathbf{H}}_{\text {spark }}^{k}(X) \longrightarrow \widehat{\mathbf{H}}_{\text {hyperspark }}^{k}(X)
$$




\section{Theorem 3.3.}

$$
\widehat{\mathbf{H}}_{\text {spark }}^{k}(X) \cong \widehat{\mathbf{H}}_{\text {hyperspark }}^{k}(X)
$$

Proof. The hyperspark complex $\left(\bar{F}^{*}, \bar{E}^{*}, \bar{I}^{*}\right)$ defined above contains the sparks: $F^{*}=\mathcal{D}^{*}(X), E^{*}=\mathcal{E}^{*}(X)$ and $I^{*}=I F^{*}(X)$ as a subspark complex (in the component $C^{0}\left(\mathcal{U}, \mathcal{D}^{\prime *}\right)$ ). Applying Proposition 1.4 completes the proof.

\section{Smooth Hypersparks.}

If a de Rham-Federer spark $a \in S^{k}(X)$ is smooth, i.e. $a \in \mathcal{E}^{k}(X) \subset \mathcal{D}^{\prime k}(X)$, then $d a=\phi$, i.e. $R=0$. In fact, $a$ is equivalent to a smooth spark if and only if the class of $R$ in $H^{k+1}(X, \mathbf{Z})$ is zero. In particular, a general spark need not be equivalent to a smooth spark. The situation is different for hypersparks. Consider the Cech-form sub-bicomplex of the full Cech-current bicomplex

$$
\bigoplus_{p, q \geq 0} C^{p}\left(\mathcal{U}, \mathcal{E}^{q}\right) \subset \bigoplus_{p, q \geq 0} C^{p}\left(\mathcal{U}, \mathcal{D}^{\prime q}\right)
$$

Definition 4.1. A hyperspark $A$ is smooth if $A \in \bigoplus_{p+q=k} C^{p}\left(\mathcal{U}, \mathcal{E}^{q}\right)$.

Note that

$$
C^{p}\left(\mathcal{U}, \mathcal{E}^{q}\right) \cap C^{p}\left(\mathcal{U}, I F^{q}\right)=\{0\}
$$

unless $q=0$, in which case:

$$
C^{p}\left(\mathcal{U}, \mathcal{E}^{0}\right) \cap C^{p}\left(\mathcal{U}, I F^{0}\right)=C^{p}(\mathcal{U}, \mathbf{Z})
$$

Consequently, each smooth hyperspark $A$ satisfies

$$
D A=\phi-R
$$

with $R \in C^{k+1}(\mathcal{U}, \mathbf{Z})$ a Čech integer cocycle.

Said differently, we are considering the sub-spark complex with $F^{k}=$ $\bigoplus_{p+q=k} C^{p}\left(\mathcal{U}, \mathcal{E}^{q}\right), E^{k}=\mathcal{E}^{k}(X) \subset C^{0}\left(\mathcal{U}, \mathcal{E}^{k}\right)$, and $I^{k}=C^{k}(\mathcal{U}, \mathbf{Z}) \subset$ $C^{k}\left(\mathcal{U}, \mathcal{E}^{0}\right)$. 


$$
\begin{array}{ccc}
\mathcal{E}^{2}(X) \subset C^{0}\left(\mathcal{U}, \mathcal{E}^{2}\right) & \longrightarrow \\
\uparrow d & \uparrow d & \uparrow \\
\mathcal{E}^{1}(X) \subset C^{0}\left(\mathcal{U}, \mathcal{E}^{1}\right) & \stackrel{-\delta}{\longrightarrow} C^{1}\left(\mathcal{U}, \mathcal{E}^{1}\right) \longrightarrow \\
\uparrow d \quad \uparrow d & \uparrow d & \\
\uparrow & \uparrow d \\
\mathcal{E}^{0}(X) \subset C^{0}\left(\mathcal{U}, \mathcal{E}^{0}\right) & \stackrel{\delta}{\longrightarrow} C^{1}\left(\mathcal{U}, \mathcal{E}^{0}\right) \stackrel{\delta}{\longrightarrow} C^{2}\left(\mathcal{U}, \mathcal{E}^{0}\right) \longrightarrow \\
\cup & \cup & \cup \\
& C^{0}(\mathcal{U}, \mathbf{Z}) \stackrel{\delta}{\longrightarrow} C^{1}(\mathcal{U}, \mathbf{Z}) \stackrel{\delta}{\longrightarrow} C^{2}(\mathcal{U}, \mathbf{Z}) \longrightarrow
\end{array}
$$

Diagram 4.1: The Smooth Čech-de Rham Bicomplex

\section{Low Degree Smooth Hypersparks.}

Degree 0: $A \in C^{0}\left(\mathcal{U}, \mathcal{E}^{0}\right), \phi \in \mathcal{E}^{1}(X), R \in C^{1}(\mathcal{U}, \mathbf{Z})$

$$
\begin{aligned}
d A_{\alpha} & =\phi \\
A_{\beta}-A_{\alpha} & =-R_{\alpha \beta} .
\end{aligned}
$$

Degree 1: $A \in C^{0}\left(\mathcal{U}, \mathcal{E}^{1}\right) \oplus C^{1}\left(\mathcal{U}, \mathcal{E}^{0}\right), \phi \in \mathcal{E}^{2}(X), R \in C^{2}(\mathcal{U}, \mathbf{Z})$

$$
\begin{aligned}
d A_{\alpha} & =\phi \\
A_{\beta}-A_{\alpha} & =-d A_{\alpha \beta} \\
A_{\beta \gamma}-A_{\alpha \gamma}+A_{\alpha \beta} & =-R_{\alpha \beta \gamma}
\end{aligned}
$$

Degree 2: $A \in C^{0}\left(\mathcal{U}, \mathcal{E}^{2}\right) \oplus C^{1}\left(\mathcal{U}, \mathcal{E}^{1}\right) \oplus C^{2}\left(\mathcal{U}, \mathcal{E}^{0}\right), \phi \in \mathcal{E}^{3}(X), R \in C^{3}(\mathcal{U}, \mathbf{Z})$

$$
\begin{aligned}
d A_{\alpha} & =\phi \in \mathcal{E}^{3}(X) \\
A_{\beta}-A_{\alpha} & =d A_{\alpha \beta} \\
A_{\alpha \beta}+A_{\beta \gamma}+A_{\gamma \alpha} & =d A_{\alpha \beta \gamma} \\
A_{\beta \gamma \delta}-A_{\alpha \gamma \delta}+A_{\alpha \beta \delta}-A_{\alpha \beta \gamma} & =-R_{\alpha \beta \gamma \delta} \in \mathbf{Z}
\end{aligned}
$$

Degree k: $A=\bigoplus_{p+q=k} A^{p, q} \in \bigoplus_{p+q=k} C^{p}\left(\mathcal{U}, \mathcal{E}^{q}\right), \phi \in \mathcal{E}^{k+1}(X), R \in$ $C^{k+1}(\mathcal{U}, \mathbf{Z})$

$$
\begin{aligned}
d A^{0, k} & =\phi \\
d A^{1, k-1}+(-1)^{k} \delta A^{0, k} & =0 \\
\vdots & \\
d A^{k, 0}-\delta A^{k-1,1} & =0 \\
\delta A^{k, 0} & =-R .
\end{aligned}
$$


For smooth hypersparks, it is natural to define equivalence using only Čechde Rham forms.

Definition 4.2. Two smooth hypersparks, $A$ and $\bar{A}$, of degree $k$, are said to be smoothly equivalent if there exist $B \in \bigoplus_{p+q=k-1} C^{p}\left(\mathcal{U}, \mathcal{E}^{q}\right)$ and $S \in$ $C^{k}(\mathcal{U}, \mathbf{Z})$ satisfying

$$
A-\bar{A}=D B+S
$$

\section{Low Degree Equivalence.}

Degree 0: $A_{\alpha}-\bar{A}_{\alpha}=S_{\alpha} \in C^{0}(\mathcal{U}, \mathbf{Z})$. Degree 1:

$$
\begin{aligned}
A_{\alpha}-\bar{A}_{\alpha} & =d B_{\alpha} \\
A_{\alpha \beta}-\bar{A}_{\alpha \beta} & =B_{\beta}-B_{\alpha}+S_{\alpha \beta}
\end{aligned}
$$

for some $B \in C^{0}\left(\mathcal{U}, \mathcal{E}^{0}\right)$ and some $S \in C^{1}(\mathcal{U}, \mathbf{Z})$.

Degree 2:

$$
\begin{aligned}
A_{\alpha}-\bar{A}_{\alpha} & =d B_{\alpha} \\
A_{\alpha \beta}-\bar{A}_{\alpha \beta} & =-B_{\beta}+B_{\alpha}+d B_{\alpha \beta} \\
A_{\alpha \beta \gamma}-\bar{A}_{\alpha \beta \gamma} & =B_{\beta \gamma}-B_{\alpha \gamma}+B_{\alpha \beta}+S_{\alpha \beta \gamma}
\end{aligned}
$$

for some $B \in C^{0}\left(\mathcal{U}, \mathcal{E}^{1}\right) \oplus C^{1}\left(\mathcal{U}, \mathcal{E}^{0}\right)$ and some $S \in C^{2}(\mathcal{U}, \mathbf{Z})$.

Let $\widehat{\mathbf{H}}_{\text {smooth }}^{k}(X)$ denote the space of smooth hypersparks under smooth equivalence. There is a natural map

$$
\widehat{\mathbf{H}}_{\text {smooth }}^{k}(X) \longrightarrow \widehat{\mathbf{H}}_{\text {hyperspark }}^{k}(X) \text {. }
$$

\section{Theorem 4.3.}

$$
\widehat{\mathbf{H}}_{\text {smooth }}^{k}(X) \cong \widehat{\mathbf{H}}_{\text {hyperspark }}^{k}(X) .
$$

Proof. Note that the smooth hypersparks form a subspark complex of the hypersparks and apply Proposition 1.4. 


\section{Grundles.}

In this section, we present grundles which are closely related (and equivalent) to smooth hypersparks, but more geometric in nature. A grundle of degree $k$ is obtained from a smooth hyperspark $A$ of degree $k$ by simply replacing the last component $A^{k, 0}$ with its exponential:

$$
g_{\alpha_{0} \ldots \alpha_{k}}=e^{2 \pi i A_{\alpha_{0} \ldots \alpha_{k}}}
$$

Thus, $g \in C^{k}\left(\mathcal{U}, \mathcal{E}_{S^{1}}\right)$ where $\mathcal{E}_{S^{1}}$ is the sheaf of smooth $S^{1}$-valued functions. The last component of the hyperspark equation:

$$
\delta A^{k, 0}=-R
$$

where $R_{\alpha_{0} \ldots \alpha_{k}} \in \mathbf{Z}$ implies that

$$
\delta g=0
$$

i.e. $g$ is a cocycle.

Definition 5.1. A grundle of degree $k$ is a pair $(A, g)$ with $g \in C^{k}\left(\mathcal{U}, \mathcal{E}_{S^{1}}\right)$, $\delta g=1$ (a cocycle) and $A \in \bigoplus_{\substack{p+q=k \\(p, q) \neq(k, 0)}} C^{p}\left(\mathcal{U}, \mathcal{E}^{q}\right)$, satisfying

$$
\begin{gathered}
d A^{0, k}=\phi \in \mathcal{E}^{k+1}(X) \\
d A^{1, k-1}+(-1)^{k} \delta A^{0, k}=0 \\
\vdots \\
d A^{k-1,1}+\delta A^{k-2,2}=0 \\
\frac{1}{2 \pi i} \frac{d g}{g}-d A^{k-1,1}=0
\end{gathered}
$$

The cocycle condition $\delta g=1$ implies that for any choice $A^{0, k}=\frac{1}{2 \pi i} \log g$ of the logarithm of the $g$, we have $\delta A^{0, k} \in C^{k+1}(\mathcal{U}, \mathbf{Z})$. Therefore, if we let $R$ denote $-\delta A^{0, k} \in Z^{k+1}(\mathcal{U}, \mathbf{Z})$, then although $R$ is not uniquely determined by $g$, the class $[R] \in H^{k+1}(\mathcal{U}, \mathbf{Z})$ is uniquely determined by $g$. That is, two different choices $A^{0, k}$ and $\bar{A}^{0, k}$ differ by $A^{0, k}-\bar{A}^{0, k}=S \in C^{0, k}(\mathcal{U}, \mathbf{Z})$ and hence $\bar{R}-R=\delta S$ represent the same class in $H^{k+1}(\mathcal{U}, \mathbf{Z})$. In other words

$$
H^{k}\left(\mathcal{U}, \mathcal{E}_{S^{1}}\right) \cong H^{k+1}(\mathcal{U}, \mathbf{Z}) \quad(k \geq 1)
$$


with $g$ representing the class in $H^{k}\left(\mathcal{U}, \mathcal{E}_{S^{1}}\right)$ and $R=-\delta A^{0, k}$ representing the class in $H^{k+1}(\mathcal{U}, \mathbf{Z})$. For $k=0$, the sequence

$$
0 \longrightarrow \mathbf{Z} \longrightarrow \mathcal{E}(X) \longrightarrow \mathcal{E}_{S^{1}}(X) \longrightarrow H^{1}(\mathcal{U}, \mathbf{Z}) \longrightarrow 0 \text { is exact. }
$$

\section{Low Degree Grundles}

Degree 0: A grundle of degree 0 is a $g \in C^{0}\left(\mathcal{U}, \mathcal{E}_{S^{1}}\right)$ satisfying the cocycle condition $\delta g=1$. That is $g \in \mathcal{E}_{S^{1}}(X)$ is just a smooth circle valued function.

Degree 1: A grundle of degree 1 consist of a cocycle $g \in Z^{1}\left(\mathcal{U}, \mathcal{E}_{S^{1}}\right)$ (i.e. $g_{i j} \in \mathcal{E}_{S^{1}}\left(\mathcal{U}_{i j}\right)$ satisfying $g_{i j} g_{j k} g_{k i}=1$.) and $A=A^{0,1} \in C^{0}\left(\mathcal{U}, \mathcal{E}^{1}\right)$ satisfying

$$
\begin{aligned}
& d A_{i}=\phi \in \mathcal{E}^{2}(X) \\
& A_{j}-A_{i}=\frac{-1}{2 \pi i} \frac{d g_{i j}}{g_{i j}} .
\end{aligned}
$$

Thus, a grundle of degree 1 is just a hermitian line bundle equipped with a local trivialization on each $\mathcal{U}_{i}$ and equipped with a unitary connection.

Degree 2: A grundle of degree 2 consists of a cocycle $g \in Z^{2}\left(\mathcal{U}, \mathcal{E}_{S^{1}}\right)$ (i.e. $g_{i j k} \in \mathcal{E}_{S^{1}}\left(\mathcal{U}_{i j k}\right)$ satisfying $\left.\delta g=1\right) A^{1,1}=\left\{A_{i j}\right\} \in C^{1}\left(\mathcal{U}, \mathcal{E}^{1}\right)$, and $A^{0,2}=\left\{A_{i}\right\} \in C^{0}\left(\mathcal{U}, \mathcal{E}^{2}\right)$ satisfying

$$
\begin{aligned}
& d A_{i}=\phi \in \mathcal{E}^{3}(X) \\
& A_{j}-A_{i}=d A_{i j} \\
& A_{i j}+A_{j k}+A_{k i}=\frac{1}{2 \pi i} \frac{d g_{i j k}}{g_{i j k}} .
\end{aligned}
$$

Thus, a grundle of degree 2 is just a gerbe with connection (see Hitchin [20]).

Definition 5.2 (Gauge Equivalence). Two grundles $(A, g)$ and $(\bar{A}, \bar{g})$ are said to be gauge equivalent if first, there exist an $h \in C^{k-1}\left(\mathcal{U}, \mathcal{E}_{S^{1}}\right)$ with $g \bar{g}^{-1}=\delta h$ (i.e. $g$ and $\bar{g}$ equivalent, via $h$, in $H^{k}\left(\mathcal{U}, \mathcal{E}_{S^{1}}\right)$ ), and second, there exist $B \in \bigoplus_{\substack{p+q=k-1 \\ q \neq 0}} C^{p}\left(\mathcal{U}, \mathcal{E}^{q}\right)$ satisfying the equations

$$
\begin{aligned}
A^{0, k}-\bar{A}^{0, k} & =d B^{0, k-1} \\
A^{1, k-1}-\bar{A}^{1, k-1} & =d B^{1, k-2}+(-1)^{k-1} \delta B^{0, k-1} \\
\vdots & \\
A^{k-1,1}-\bar{A}^{k-1,1} & =\frac{1}{2 \pi i} \frac{d h}{h}-\delta B^{k-2,1},
\end{aligned}
$$




\section{Low Degree Gauge Equivalence}

Degree 0: Two grundles $g$ and $\bar{g} \in \mathcal{E}_{S^{1}}(X)$ are gauge equivalent if they are equal.

Degree 1: Two grundles $(A, g)$ and $(\bar{A}, \bar{g})$ are gauge equivalent if there exist $h \in C^{0}\left(\mathcal{U}, \mathcal{E}_{S^{1}}\right)$ with $g_{i j} \bar{g}_{i j}^{-1}=h_{i} / h_{j}$ and $A_{i}-\bar{A}_{i}=\frac{1}{2 \pi i} \frac{d h_{i}}{h_{i}}$. Thus, equivalence classes of degree 1 grundles are the same as equivalence classes of unitary line bundles with unitary connection.

Degree 2: Grundles $(A, g)$ and $(A, \bar{g})$ (i.e. gerbes with connection) are gauge equivalent if there exist a cochain $h \in C^{1}\left(\mathcal{U}, \mathcal{E}_{S^{1}}\right)$ and $B \in C^{1}\left(\mathcal{U}, \mathcal{E}^{1}\right)$ satisfying

$$
\begin{aligned}
A_{i}-\bar{A}_{i} & =d B_{i} \\
A_{i j}-\bar{A}_{i j} & =\frac{1}{2 \pi i} \frac{d h_{i j}}{h_{i j}}-B_{j}+B_{i} \\
g_{i j k} \bar{g}_{i j k}^{-1} & =h_{i j} h_{j k} h_{k i}
\end{aligned}
$$

Remark 5.3. Let $\widehat{\mathbf{H}}_{\text {grundle }}^{k}(X)$ denote the gauge equivalence classes of degree $k$ grundles on $X$. It is straightforward to check that there is an isomorphism

$$
\widehat{\mathbf{H}}_{\mathrm{smooth}}^{k}(X) \cong \widehat{\mathbf{H}}_{\text {grundle }}^{k}(X)
$$

induced by exponentiation of the last term as in (5.1).

\section{Cheeger-Simons Sparks.}

Consider smooth singular (integral) chains $C_{q}(X)$ on $X$ and let $C_{\mathbf{Z}}^{q}(X)$ $=\operatorname{Hom}\left(C_{q}(X), \mathbf{Z}\right)$ denote the space of integer cochains of degree $q$. Let $C_{\mathbf{R}}^{q}(X) \supset C_{\mathbf{Z}}^{q}(X)$ denote the vector space of real cochains on $X$. Each smooth form $\phi \in \mathcal{E}^{q}(X)$ determines, via integration, a real cochain. This cochain uniquely determines the form $\phi$, i.e. if a form $\phi$ integrates to zero over all singular chains, then $\phi=0$. Also, it is easy to see that $\mathcal{E}^{q}(X) \cap C_{\mathbf{Z}}^{q}(X)=0, q=1,2, \ldots$ and $\mathcal{E}^{0}(X) \cap C_{\mathbf{Z}}^{0}(X)=\mathbf{Z}$. Thus, $E^{*}=\mathcal{E}^{*}(X), F^{*}=C_{\mathbf{R}}^{*}(X)$ and $I^{*}=C_{\mathbf{Z}}^{*}(X)$ form a spark complex. The associated sparks are called "Cheeger-Simons sparks", that is, we have the following. 
Definition 6.1. A Cheeger-Simons spark of degree $k$ on $X$ is a real cochain $a \in C_{\mathbf{R}}^{k}(X)$ with the property that

$$
d a=\phi-r
$$

with $\phi \in \mathcal{E}^{k+1}(X)$ a smooth form and $r \in C_{\mathbf{Z}}^{k+1}(X)$ an (integer) cochain. Two such sparks are equivalent if they differ by a real cochain of the form $d b+s$ where $b \in C_{\mathbf{R}}^{k-1}(X)$ and $s \in C_{\mathbf{Z}}^{k}(X)$. Let $\widehat{\mathbf{H}}_{\mathrm{CS}}^{k}$ denote the space of Cheeger-Simons spark classes.

Theorem 6.2. There exists a natural isomorphism

$$
\widehat{\mathbf{H}}_{\mathrm{smooth}}^{k}(X) \cong \widehat{\mathbf{H}}_{C S}^{k}(X) \text {. }
$$

Proof. We must show that the spark complex of smooth hypersparks

$$
E^{*}=\mathcal{E}^{*}(X), F^{*}=\bigoplus_{p+q=*} C^{p}\left(\mathcal{U}, \mathcal{E}^{q}\right), \text { and } I^{*}=C^{*}(\mathcal{U}, \mathbf{Z})
$$

and the spark complex of Cheeger-Simons sparks

$$
E^{*}=\mathcal{E}^{*}(X), F^{*}=C_{\mathbf{R}}^{*}(X), \text { and } I^{*}=C_{\mathbf{Z}}^{*}(X)
$$

are compatible. Let $C_{\mathbf{R}}^{q}$ denote the sheaf of germs of real $q$-cochains and let $C_{\mathbf{Z}}^{q}$ denote the subsheaf of (integer) cochains. The spark complex with

$$
\begin{aligned}
& \bar{E}^{*}=\mathcal{E}^{*}(X), \\
& \bar{F}^{*}=\bigoplus_{p+q=*} C^{p}\left(\mathcal{U}, \mathcal{C}_{\mathbf{R}}^{q}\right), \quad \text { and } \\
& \bar{I}^{*}=\bigoplus_{p+q=*} C^{p}\left(\mathcal{U}, \mathcal{C}_{\mathbf{Z}}^{q}\right)
\end{aligned}
$$

contains both of the previous spark complexes. This larger "cochain hyperspark complex" and Proposition 1.4 complete the proof.

\section{Differential Characters.}

In their fundamental paper [3], Cheeger and Simons defined the space of differential characters of degree $k$ to be the group

$$
\begin{array}{r}
\operatorname{DiffChar}^{k}(X)=\left\{h \in \operatorname{Hom}\left(Z_{k}(X), \mathbf{R} / \mathbf{Z}\right): d h \equiv \phi(\bmod \mathbf{Z})\right. \\
\left.\quad \text { for some } \phi \in \mathcal{E}^{k+1}(X)\right\} .
\end{array}
$$

where $Z_{k}(X) \subset C_{k}(X)$ denotes the group of smooth singular $k$-cycles with Z-coefficients. 
Proposition 7.1. For any manifold $X$, there is a natural isomorphism

$$
\widehat{\mathbf{H}}_{\mathrm{CS}}^{k}(X) \cong \operatorname{DiffChar}^{\mathrm{k}}(\mathrm{X})
$$

Proof. Suppose $a \in C_{\mathbf{R}}^{k}(X)$ is a Cheeger-Simons spark. Define $h_{a}$ to be $a$ restricted to $Z_{k}(X)$ modulo $\mathbf{Z}$. If $a=d b+s$ represents the zero spark class, then $h_{a}(c)=d b(c)+s(c)=s(c) \in \mathbf{Z}$ for every $c \in Z_{k}(X)$. Thus, $h_{a}$ depends only on the spark class $\widehat{a}$.

Onto: Now, given $h \in \operatorname{Hom}\left(Z_{k}(X), \mathbf{R} / \mathbf{Z}\right)$, we may lift to a cochain $a \in$ $C_{\mathbf{R}}^{k}(X)$. Moreover, $d h \equiv \phi(\bmod \mathbf{Z})$ is equivalent to $d a-\phi$ being $\mathbf{Z}$-valued on $C_{k+1}(X)$, i.e. $d a-\phi=-r \in C_{\mathbf{Z}}^{k+1}(X)$ is an integer cochain. This proves that $a$ is a Cheeger-Simons spark.

One-to-One: Suppose $a \in C_{\mathbf{R}}^{k}(X)$ is a Cheeger-Simons spark (i.e. $d a=$ $\phi-r$ with $\phi \in \mathcal{E}^{k+1}(X) \subset C_{\mathbf{R}}^{k+1}(X)$ and $r \in C_{\mathbf{Z}}^{k+1}(X)$. Let $h_{a}$ denote the induced differential character. Suppose $h_{a}=0$, that is, suppose

$$
a: Z_{k}(X) \rightarrow \mathbf{Z}
$$

is integer-valued. Pick an extension $s \in \operatorname{Hom}\left(C_{k}(X)\right.$, Z), i.e. $s \in C_{\mathbf{Z}}^{k}(X)$ and $a-s$ vanishes on $Z_{k}(X)$. Consequently, $a-s=d b$ for some $b \in C_{\mathbf{R}}^{k-1}(X)$, i.e. the Cheeger-Simons spark $a$ is equivalent to zero.

\section{Holonomy Maps.}

Recall now that every $C^{\infty}$ singular integral $k$-chain $c \in C_{k}(X)$ determines a current $\widetilde{c} \in \mathcal{D}^{\prime k}(X)$ by integration of forms over $c$. The image of this map, denoted $\widetilde{C}_{k}(X) \subset \mathcal{D}^{\prime k}(X)$, is the de Rham group of current chains in dimension $k$. There is a short exact sequence of chain complexes

$$
0 \rightarrow N_{k}(X) \rightarrow C_{k}(X) \stackrel{\rho}{\longrightarrow} \widetilde{C}_{k}(X) \rightarrow 0
$$

and the map $\rho(c)=\widetilde{c}$ induces an isomorphism in homology (cf. [5]). The elements of $N_{k}(X)$ will be called null chains. To get some feeling for this, note that integration over a compact oriented $k$-dimensional submanifold $\Sigma \subset X$ defines an element $[\Sigma] \in \widetilde{C}_{k}(X)$. Every smooth triangulation of $\Sigma$ yields a chain $c \in C_{k}(X)$ with $\rho(c)=[\Sigma]$.

Let $\widetilde{Z}_{k}(X)$ denote the group of cycles in $\widetilde{C}_{k}(X)$. We define the set of holonomy maps of degree $k$ to be the group

$$
\begin{array}{r}
\mathbf{H o l}^{k}(X)=\left\{H \in \operatorname{Hom}\left(\widetilde{Z}_{k}(X), \mathbf{R} / \mathbf{Z}\right): d H \equiv \phi(\bmod \mathbf{Z})\right. \\
\text { for some } \left.\phi \in \mathcal{E}^{k+1}(X)\right\} .
\end{array}
$$


The form $\phi$ is called the associated curvature form. Here $d H(c)=H(\partial c)$ by definition. Of course, if $H \in \mathbf{H o l}^{k}(X)$, then $h=H \circ \rho \in \operatorname{DiffChar}^{k}(X)$.

Theorem 8.1. Every differential character $h \in \operatorname{Hom}\left(Z_{k}(X), \mathbf{R} / \mathbf{Z}\right)$ descends to a homomorphism $H \in \operatorname{Hom}\left(\widetilde{Z}_{k}(X), \mathbf{R} / \mathbf{Z}\right)$, i.e. it can be written in the form $h=H \circ \rho$. This yields a natural isomorphism

$$
\operatorname{DiffChar}^{\mathrm{k}}(\mathrm{X})=\mathbf{H o l}^{\mathrm{k}}(\mathrm{X})
$$

Proof. The pull-back map $\operatorname{Hol}^{k}(X) \longrightarrow \operatorname{DiffChar}^{k}(X)$ given by $H \mapsto H \circ \rho$, is obviously injective. Therefore, the isomorphism follows from the first assertion. To prove this, let $h \in \operatorname{DiffChar}^{k}(X)$ be any differential character. By definition $d h=\phi$ for some smooth $(k+1)$-form $\phi$. Now, it suffices to show that for any pair $c_{1}, c_{2} \in Z_{k}(X)$ satisfying $\widetilde{c}_{1}=\widetilde{c}_{2}$, there exists a null chain $B$ with $c_{1}-c_{2}=d B$ because in this case

$$
h\left(c_{1}\right)-h\left(c_{2}\right)=h(d B)=\int_{B} \phi=0 .
$$

Note that $a=c_{1}-c_{2}$ is a null cycle. Furthermore, the homology class of $a$ in $C_{*}(X)$ must be zero since $\widetilde{a}=0$ and the map $\rho(c) \equiv \widetilde{c}$ in $(7.1)$ is injective in homology. Hence, there exists $b \in C_{k+1}(X)$ with $d b=a$, and we have $\widetilde{d b}=\widetilde{a}=0$. Now, since the map $\rho$ is surjective in homology, we can write $\widetilde{b}=\widetilde{z}+d \widetilde{e}$ where $d z=0$. Set $B=b-z-d e$. Then, $B$ is a null chain with $d B=a=c_{1}-c_{2}$ as desired.

\section{Grundle holonomy.}

Combining Remark 5.3, Theorem 6.2, Proposition 7.1 and Theorem 8.1, we see that every grundle $(A, g)$ of degree $k$ induces a homomorphism

$$
h_{(A, g)}: \widetilde{Z}_{k}(X) \longrightarrow \mathbf{R} / \mathbf{Z}=S^{1}
$$

called the holonomy of $(A, g)$. It depends only on the gauge-equivalence class of the grundle. (By Theorem 4.7, every hyperspark also has such a holonomy homomorphism.)

In particular, let $M$ be a compact connected oriented manifold of dimension $k(\partial M=\emptyset)$ and $f: M \rightarrow X$ a smooth map. Then by Theorem 8.2, the holonomy of $(A, g)$ on $M$

$$
h_{(A, g)}(M) \in S^{1}
$$


is well-defined (i.e. independent of the choice of triangulation of $M$ ). It can be computed as follows. The induced grundle $f^{*}(A, g)$ represents a class in $\widehat{\mathbf{H}}_{\text {grundle }}^{k}(M)=H^{k}(M ; \mathbf{R}) / H^{k}(M ; \mathbf{Z})=\operatorname{Hom}\left(H_{k}(M ; \mathbf{Z}), S^{1}\right)=$ $\operatorname{Hom}\left(\mathbf{Z} \cdot[M], S^{1}\right)$ by the diagram at the end of Section 2 . Then, $h_{(A, g)}(M)=$ $\left[f^{*}(A, g)\right]([M])$.

What is most interesting is to find intrinsic geometric definitions of the holonomy over such cycles $M$ and to find combinatorial formulae for $h_{(A, g)}(M)$ in terms of the grundle data. A basic example of this occurs in degree 1 where equivalence classes of grundles coincide with gauge equivalence classes of complex line bundles with unitary connection. Here, the holonomy around a closed loop $\gamma$ coincides with the rotation angle obtained by parallel translation around $\gamma$ (the classical holonomy) [3]. This is expressed in the combinatorial grundle formula derived below.

We shall systematically derive formulae for grundles of all degrees.

The general procedure is as follows. Consider a grundle of degree $k$ presented by a smooth hyperspark $A$. (That is, we choose logarithms $A_{\alpha_{0} \ldots \alpha_{k}}$ for the $g_{\alpha_{0} \ldots \alpha_{k}}$ as in (5.1).) Let $h$ be a Cheeger-Simons spark which is equivalent to $A$ in the cochain hyperspark complex. Then, the holonomy $h_{A}: Z_{k}(X) \rightarrow \mathbf{R} / \mathbf{Z}$ is given by

$$
h_{A}(z) \equiv h(z) \quad(\bmod \mathbf{Z})
$$

The idea now is to use the equivalence between $A$ and $h$ to generate the formula.

Degree 0: Here, a 0-grundle is given by $g_{\alpha}=\exp \left(2 \pi i A_{\alpha}\right)$ on $U_{\alpha} \in \mathcal{U}$. Let $h \in C_{\mathbf{R}}^{0}(X)$ be an equivalent Cheeger-Simons spark. Then, $A_{\alpha}-h=S_{\alpha}$ in $U_{\alpha}$ for some $S \in C^{0}\left(\mathcal{U}, C_{\mathbf{Z}}^{0}\right)$. We conclude that the holonomy on the zero-cycle $x \in X$ can be written as

$$
h_{A}(x) \equiv A_{\alpha}(x) \quad(\bmod \mathbf{Z})
$$

where $x \in U_{\alpha}$. Of course, as noted in Section 5, a grundle of degree 0 is just a smooth map $g: X \rightarrow S^{1}$, and its holonomy at $x$ is seen to be $g(x) \in S^{1}$. This is the "intrinsic" definition of holonomy in degree 0 .

Degree 1: Let $A=\left(\left\{A_{\alpha \beta}\right\},\left\{A_{\alpha}\right\}\right) \in C^{1}\left(\mathcal{U}, \mathcal{E}^{0}\right) \oplus C^{0}\left(\mathcal{U}, \mathcal{E}^{1}\right)$ be a smooth hyperspark representing a 1-grundle as above, and let $h \in C_{\mathbf{R}}^{1}(X)$ be an equivalent Cheeger-Simons spark. The elements $A$ and $h$ both lie in $C^{1}\left(\mathcal{U}, C_{\mathbf{R}}^{0}\right) \oplus C^{0}\left(\mathcal{U}, C_{\mathbf{R}}^{1}\right)$ where $h=\left(\left\{h_{\alpha, \beta}\right\},\left\{h_{\alpha}\right\}\right)=\left(0,\left.h\right|_{U_{\alpha}}\right)$. The equivalence of $A$ and $h$ means there exist elements $a \in C^{0}\left(\mathcal{U}, C_{\mathbf{R}}^{0}\right)$ and 
$S \in C^{1}\left(\mathcal{U}, C_{\mathbf{Z}}^{0}\right) \oplus C^{0}\left(\mathcal{U}, C_{\mathbf{Z}}^{1}\right)$ with

$$
\begin{aligned}
d a_{\alpha} & =A_{\alpha}-h+S_{\alpha} \\
a_{\beta}-a_{\alpha} & =A_{\alpha \beta}+S_{\alpha \beta}
\end{aligned}
$$

Let $\gamma \in \mathcal{Z}_{1}(X)$ be a closed loop and write $\gamma=\sum_{\alpha=1}^{N} \gamma_{\alpha}$ where $\gamma_{1}, \ldots, \gamma_{N}$ are successive 1-simplices subordinate to elements $U_{1}, \ldots, U_{N}$ of the covering. Set $V_{\alpha, \alpha+1}=\gamma_{\alpha+1} \cap \gamma_{\alpha}$ with indices taken $\bmod N$. Then, using Theorem 8.2 and calculating with Equation (9.1), we find

$$
\begin{aligned}
h_{A}(\gamma) \equiv \sum_{\alpha=1}^{N} h\left(\gamma_{\alpha}\right) & \equiv \sum_{\alpha=1}^{N}\left\{\int_{\gamma_{\alpha}} A_{\alpha}+a_{\alpha}\left(d \gamma_{\alpha}\right)\right\} \quad(\bmod \mathbf{Z}) \\
& \equiv \sum_{\alpha=1}^{N}\left\{\int_{\gamma_{\alpha}} A_{\alpha}+a_{\alpha}\left(V_{\alpha, \alpha+1}-V_{\alpha-1, \alpha}\right)\right\} \quad(\bmod \mathbf{Z}) \\
& \equiv \sum_{\alpha=1}^{N} \int_{\gamma_{\alpha}} A_{\alpha}-\sum_{\alpha=1}^{N} A_{\alpha, \alpha+1}\left(V_{\alpha, \alpha+1}\right) \quad(\bmod \mathbf{Z}) .
\end{aligned}
$$

Remark 9.1. Suppose that our grundle data $\left\{A_{j}\right\}$ and $\left\{g_{i, j}\right\}$, with $g_{i j}=$ $\exp \left(2 \pi i A_{i j}\right)$, correspond to the line bundle with connection $(L, D)$. Then, $A_{j}$ represents the connection 1 -form with respect to a nowhere vanishing section $\sigma_{j}$ of $\left.L\right|_{U_{j}}$, and $g_{i j}$ is the change of trivialization on $U_{i} \cap U_{j}$. One has that $D \sigma_{j}=i A_{j} \otimes \sigma_{j}$, and a section $\sigma=f_{j} \sigma_{j}$ is parallel along the arc $I_{j} \subset U_{j}$ iff $d f_{j}+i f_{j} A_{j}=0$ on $I_{j}$. Therefore, the effect of parallel translation in $L$ once around the loop $\gamma$, is given by the formula:

$$
h_{A}(\gamma) \equiv \sum_{j=1}^{N} \int_{\gamma_{j}} A_{j}-\sum_{j=1}^{N} \int_{V_{j}} A_{j, j+1} \quad(\bmod \mathbf{Z})
$$

which we derived above. The first integrals represent parallel translation along $I_{j}$ in the given frame; the second integrals represent the change of frame at the vertex.

Degree 2: Let $A=\left(\left\{A_{\alpha \beta \gamma}\right\},\left\{A_{\alpha \beta}\right\},\left\{A_{\alpha}\right\}\right) \in C^{2}\left(\mathcal{U}, \mathcal{E}^{0}\right) \oplus C^{1}\left(\mathcal{U}, \mathcal{E}^{1}\right) \oplus$ $C^{0}\left(\mathcal{U}, \mathcal{E}^{2}\right)$ be a smooth hyperspark representing the 2 -grundle (or gerbe with connection) as above, and let $h \in C_{\mathbf{R}}^{2}(X)$ be an equivalent Cheeger-Simons spark. Equivalence in the cochain hyperspark complex means that there exist elements $a \in C^{1}\left(\mathcal{U}, C_{\mathbf{R}}^{0}\right) \oplus C^{0}\left(\mathcal{U}, C_{\mathbf{R}}^{1}\right)$ and $S \in C^{2}\left(\mathcal{U}, C_{\mathbf{Z}}^{0}\right) \oplus C^{1}\left(\mathcal{U}, C_{\mathbf{Z}}^{1}\right) \oplus$ 
$C^{0}\left(\mathcal{U}, C_{\mathbf{Z}}^{2}\right)$ with

$$
\begin{aligned}
d a_{\alpha} & =A_{\alpha}-h+S_{\alpha} \\
a_{\beta}-a_{\alpha}-d a_{\alpha \beta} & =A_{\alpha \beta}+S_{\alpha \beta} \\
a_{\alpha \beta}+a_{\beta \gamma}+a_{\gamma \alpha} & =A_{\alpha \beta \gamma}+S_{\alpha \beta \gamma}
\end{aligned}
$$

Suppose now that $\Sigma$ is a compact oriented surface without boundary in $X$. We assume that $\Sigma$ is provided with a smooth cell structure $T^{*}$ dual to some triangulation $T$ of $\Sigma$. The 2-cells of $T^{*}$ are polygons defined by taking the stars of the vertices of $T$ in its first barycentric subdivision. Each vertex of $T^{*}$ meets exactly three edges and three 2-cells. By taking $T$ fine enough, we may assume each 2-cell $P$ of $T^{*}$ is contained in some $U_{\alpha} \in \mathcal{U}$. We choose one such $U_{\alpha} \supset P$ and label $P$ as $P_{\alpha}$. Each $P_{\alpha}$ is oriented by the orientation of $\Sigma$. Each edge $E$ is contained in exactly two faces $P_{\alpha}$ and $P_{\beta}$. We label $E$ as $E_{\alpha \beta}$ and orient $E_{\alpha \beta}$ as the boundary of $P_{\alpha}$. (Thus, $E_{\beta \alpha}$ is oriented oppositely, as the boundary of $P_{\beta}$.) Each vertex $V$ meets exactly three edges and three faces and is thereby labelled $V_{\alpha \beta \gamma}$. Using the Equations (9.3) and arguing as in the degree -1 case, we find that the holonomy of our gerbe with connection $A$ on $\Sigma$ is given by the formula:

$$
h_{A}(\Sigma) \equiv \sum_{\alpha} \int_{P_{\alpha}} A_{\alpha}-\sum_{\alpha \beta} \int_{E_{\alpha \beta}} A_{\alpha \beta}+\sum_{\alpha \beta \gamma} \int_{V_{\alpha \beta \gamma}} A_{\alpha \beta \gamma} \quad(\bmod \mathbf{Z})
$$

Degree k: Let $A \in C^{k}\left(\mathcal{U}, \mathcal{E}^{0}\right) \oplus \cdots \oplus C^{0}\left(\mathcal{U}, \mathcal{E}^{k}\right)$ be a smooth hyperspark and $h \in C_{\mathbf{R}}^{k}(X)$ an equivalent Cheeger-Simons spark. Then as above, there exist elements $a \in C^{*}\left(\mathcal{U}, C_{\mathbf{R}}^{*}\right)$ and $S \in C^{*}\left(\mathcal{U}, C_{\mathbf{Z}}^{*}\right)$ such that

$$
D a=A-h+S
$$

where $D$ is the total differential as in Section 3. Now, let $M$ be a compact oriented submanifold of dimension $k$ in $X$. We suppose $M$ is given a smooth cell structure which is dual to a triangulation and for which each $k$-cell $e_{\alpha}$ is contained in an open set $U_{\alpha} \in \mathcal{U}$. Each $(k-\ell)$-cell is contained in exactly $\ell+1 k$-cells and is thereby labelled $e_{\alpha_{0} \ldots \alpha_{\ell}}$. The cell $e_{\alpha}$ is oriented by $M$. Inductively, the cell $e_{\alpha_{0} \ldots \alpha_{\ell}}$ is oriented as part of the boundary of $e_{\alpha_{0} \ldots \alpha_{\ell-1}}$. Then, proceeding as above, one finds that

$$
h_{A}(M) \equiv \sum_{\ell=0}^{k}(-1)^{\ell} \sum_{\alpha_{0} \ldots \alpha_{\ell}} \int_{e_{\alpha_{0} \ldots \alpha_{\ell}}} A_{\alpha_{0} \ldots \alpha_{\ell}} \quad(\bmod \mathbf{Z})
$$

where the $\ell^{\text {th }}$ sum is over the $(k-\ell)$-cells in the complex. 
Remark 9.2 (Holonomy via de Rham-Federer Sparks). Let $A$ and $M$ be as above and suppose $A$ is equivalent to a de Rham-Federer spark $a \in \mathcal{D}^{\prime k}(X)$. By changing $a$ by a boundary $d b, b \in \mathcal{D}^{\prime k-1}(X)$, we may assume that $a$ is smooth on $M$ (see [17, Prop. 4.2].) If $a$ is a de Rham-Federer spark which is smooth, then $a$ is also a smooth hyperspark with only one non-zero component, namely of bidegree $0, k$. Consequently, (9.6) reduces to:

$$
h_{A}(M) \equiv \int_{M} a \quad(\bmod \mathbf{Z})
$$

This direct connection between de Rham-Federer sparks and differential characters given by (9.7) was the basis for showing $\widehat{\mathbf{H}}_{\text {spark }}^{k}(X) \cong$ $\widehat{\mathbf{H}}_{\text {Diff Char }}^{k}(X)$ in [17].

\section{Further Spark Complexes.}

There are many interesting homological spark complexes which are not treated in this paper. We sketch two quick examples.

Combinatorial Sparks. Suppose $X$ is a manifold provided with a smooth triangulation (or cubulation) $\mathcal{T}$. Consider the family of all barycentric subdivisions (or dyadic subdivisions) of $\mathcal{T}$, and let $C_{k}(\mathcal{T})$ denote the abelian group generated by the oriented $k$-simplices (or $k$-cubes) belonging to these subdivisions, modulo the relation $\sigma=-\sigma^{*}$ where ${ }^{*}$ indicates the reversed orientation. Then, we can define

(10.1)

$F^{*}=\operatorname{Hom}\left(C_{*}(\mathcal{T}), \mathbf{R}\right), \quad E^{*}=\operatorname{Image}\left(\mathcal{E}^{*}(X) \rightarrow F^{*}\right), \quad I^{*}=\operatorname{Hom}\left(C_{*}(\mathcal{T}), \mathbf{Z}\right)$.

Note that because of the subdivisions, the differential forms $\mathcal{E}^{*}(X)$ inject into $F^{*}$. From this and the fact that $C_{*}(\mathcal{T})$ computes the integral homology of $X$, we have that $H^{*}(X ; \mathbf{R}) \cong H^{*}\left(F^{*}\right) \cong H^{*}\left(E^{*}\right)$. Using the subdivisions, we also see that $E^{k} \cap I^{k}=\{0\}$ for $k>0$. Hence, (10.1) is a homological spark complex. Since $H^{*}\left(I^{*}\right) \cong H^{*}(X ; \mathbf{Z})$, one sees that this complex is compatible with the Cheeger-Simons sparks. Hence, the associated group of homological spark classes $\widehat{\mathbf{H}}^{k}$ coincide with the differential characters $\widehat{\mathbf{H}}_{\text {Diff Char }}^{k}(X)$. When $\mathcal{T}$ is a cubulation, this gives a direct approach to Cheeger's $*$-product [2].

Current Cochain Sparks. Suppose $X$ is a manifold and let $\widetilde{C}_{*}(X)$ denote de Rham's complex of current chains discussed in Section 8. Then, we can define the complex of current cochains by $F^{k}=\widetilde{C}^{k}(X)=\operatorname{Hom}\left(\widetilde{C}_{k}(X), \mathbf{R}\right)$ 
with $I^{k}=\operatorname{Hom}\left(\widetilde{C}_{k}(X), \mathbf{Z}\right)$ the integral current cochains. The differential forms $E^{*}=\mathcal{E}^{*}(X)$ clearly inject into $F^{*}$, and since $\widetilde{C}_{*}(X)$ computes the integral homology of $X$, we have that $H^{*}(X ; \mathbf{R}) \cong H^{*}\left(F^{*}\right) \cong H^{*}\left(E^{*}\right)$. Evidently, $E^{k} \cap I^{k}=\{0\}$ for $k>0$, and we have a homological spark complex.

As we saw in Section 8, the natural map from $F^{*}$, current cochains, to $\bar{F}^{*}$, cochains, is not injective. However, as noted in Remark 1.7, Proposition 1.4 can be easily modified to include this case, proving that current cochain spark class are isomorphic to Cheeger-Simons spark classes.

Just as it was straightforward to show (cf. Proposition 7.1) that Cheeger-Simons spark classes are isomorphic to differential characters, it is straightforward to show that current cochain spark classes are isomorphic to $\mathbf{H o l}(X)$. This provides another proof of Theorem 8.2.

\section{Applications and Examples.}

To give the reader a feeling of the abundance and usefulness of spark complexes, we briefly sketch a number of examples and applications. The emphasis is on evaluating which of the approaches to the $(\mathbf{R}, \mathbf{Z})$ theory is best suited for the particular application or example being considered. Frequently, multiple points of view are enlightening.

Ring structure. One of the deep and most useful features of $(\mathbf{R}, \mathbf{Z})$-spark classes is the existence of a natural graded ring structure $*: \widehat{\mathbf{H}}^{*}(X) \times$ $\widehat{\mathbf{H}}^{*}(X) \rightarrow \widehat{\mathbf{H}}^{*}(X)$. The difficulty of establishing this product depends greatly on the formulation (i.e. spark complex) chosen to represent characters. The original construction by Cheeger and Simons [3, 2] was quite non-trivial due to the difference between the wedge-product of differential forms and the cup-product of the cochains they define. If one takes a grundle approach or the approach of (even smooth) hypersparks, the existence of this product seems something of a miracle. In the de Rham-Federer spark approach, there is a simple and quite useful formula for this product $[17,8]$ which holds for generic sparks in any pair of spark classes. It is established using transversality theory for flat and rectifiable currents.

These remarks apply to the $(\mathcal{O}, \mathbf{Z})$-spark classes discussed in [15].

We see that for this ring structure, the existence of many different approaches is quite useful. Another example is the following.

Functoriality. Any smooth mapping $f: X \rightarrow Y$ between manifolds induces a ring homomorphism $f^{*}: \widehat{\mathbf{H}}^{*}(Y) \rightarrow \widehat{\mathbf{H}}^{*}(X)$ compatible with $\delta_{1}$ and $\delta_{2}$. This assertion is evident from say the Cheeger-Simons or the holonomy approach 
to characters, and also in the setting of smooth hypersparks. However, it is far from clear in many other approaches since, for example, the pull-back of currents is not universally defined. Here, the ability to switch from one theory to the other is quite useful.

Gysin maps and Thom homomorphisms. To a smooth proper submersion $f: X \rightarrow Y$ between oriented manifolds, there is associated a Gysin mapping $f_{*}: \widehat{\mathbf{H}}^{*}(X) \rightarrow \widehat{\mathbf{H}}^{*}(Y)$ compatible with $\delta_{1}$ and $\delta_{2}$. This is completely evident from the de Rham-Federer viewpoint (cf. [17, Section 10]), but rather mysterious from many other points of view. Similarly, using sparks, one can naturally define Thom homomorphisms [17, Section 9] which are not so evident in other formulations.

Secondary (refined) characteristic classes. Working from their viewpoint, Cheeger and Simons developed a full theory of characteristic classes associated to principal bundles with connection [3]. It simultaneously refined both Chern-Weil theory and the theory of integral characteristic classes, and it gave new invariants for flat bundles and foliations. Their elegant arguments involved functorialty and the existence of classifying spaces for bundles with connection.

These refined classes can also be defined from the de Rham-Federer spark viewpoint. The construction involves a choice of section or bundle map $\alpha$ and yields an $L_{\text {loc }}^{1}$-transgression form $T$ with $d T=\varphi-S(\alpha)$ where $\varphi$ is a characteristic form and $S(\alpha)$ is a rectifiable current measuring certain singularities of $\alpha$. The secondary Chern and Pontrjagin classes can be systematically developed in this way. The advantage here is that singularities of geometric problems explicitly enter the picture.

Example: The Euler Spark. An understanding of this case provides geometric motivation for the spark equation and spark equivalence. Suppose $E$ is a real oriented vector bundle. Equipping $E$ with a metric connection enables one to compute an Euler (Pfaff) form $\chi$ for $E$. Equipping $E$ with a section $s$ whose zero set is reasonable (say $s$ has simple zeros or more generally $s$ is atomic) provides a divisor or zero current $\operatorname{Div}(s)$. In fact, there is a canonically defined $L_{\text {loc }}^{1}$ spark $\sigma$ satisfying the local Gauss-Bonnet equation (the spark equation):

$$
d \sigma=\chi-\operatorname{Div}(s) \quad \text { on } X
$$

See [19] for the details of this discussion. The explicit formula for $\sigma$ as a fiber integral leads one to compare two sparks $\sigma$ and $\sigma^{\prime}$ generated by two sections $s$ and $s^{\prime}$ by considering the section $\gamma=t s+(1-t) s^{\prime}$ of the bundle 
$\widetilde{E}$, equal to $E$ pulled back over $\mathbf{R} \times X$. Now, a Stokes theorem argument yields the spark comparison formula

$$
\sigma-\sigma^{\prime}=d L+R \quad \text { on } X .
$$

where $L$ is a fiber integral and hence an $L_{\text {loc }}^{1}$ form, and where $R$ is the pushforward of the divisor of $\gamma$ (cut off on $0 \leq t \leq 1$ ), and hence, is a rectifiable current.

Note that taking the exterior derivative of both sides yields the divisor comparison formula

$$
\operatorname{Div}\left(s^{\prime}\right)-\operatorname{Div}(s)=d R .
$$

In summary, the refined Euler class $\hat{\chi} \in \widehat{H}^{n}(X)$ of a bundle with metric connection is the spark class made up of the Euler sparks $\sigma$. Its divisor class is the euler class $\chi$ of $E$ in $H^{n}(X, \mathbf{Z})$ made up of the divisors $\operatorname{Div}(s)$, its curvature is the Euler Pfaff form $\chi$, and the spark equation is just the local Gauss-Bonnet equation above.

Flat sparks. A Flat spark is any representative of a class $c \in \widehat{\mathbf{H}}^{k}(X)$ whose curvature form vanishes, i.e. $\phi \in \operatorname{ker} \delta_{1}=H^{k}\left(X, S^{1}\right)$. The grundle point of view is very natural in this case. Moreover, the Cech representation of $H^{k}\left(X, S^{1}\right)$ shows that there exists a grundle representative of the form $(A, g)$ with $A=0$ and with $g \in \mathcal{Z}^{k}\left(\mathcal{U}, S^{1}\right)$ actually taking locally constant values in $S^{1}$.

The holonomy point of view is also natural in this case. A holonomy map is flat if it vanishes on boundaries. This induces a homomorphism from $H_{k}(X ; \mathbf{Z}) \cong H^{n-k}(X ; \mathbf{Z})$ to $S^{1}$, so that $\mathbf{H o l}_{\text {flat }}^{k}(X) \cong \operatorname{Hom}\left(H^{n-k}(X ; \mathbf{Z}), S^{1}\right)$. Thus, the isomorphism

$$
\operatorname{Hol}_{\text {flat }}^{k}(X) \cong H^{k}\left(X ; S^{1}\right)
$$

is equivalent to

$$
\operatorname{Hom}\left(H^{n-k}(X ; \mathbf{Z}), S^{1}\right) \cong H^{k}\left(X ; S^{1}\right)
$$

which is one way to state the classical Poincaré Duality Theorem over $\mathbf{Z}$. The same discussion applies to flat differential characters.

The de Rham-Federer point of view is natural once the isomorphism

$$
H^{k}\left(X ; S^{1}\right) \cong \frac{\left\{a \in \mathcal{D}^{\prime k-1}(X): d a \in \operatorname{Rect}^{k+1}(X)\right\}}{d \mathcal{D}^{\prime k-1}(X)+\operatorname{Rect}^{k}(X)}
$$

is established. (See [17, pg 8] for a proof.) One considers all sparks $a$ satisfying the spark equation $d a=\phi-R$ with $\phi=0$. Note that spark equivalence is the same as the equivalence relation for $H^{1}\left(X, S^{1}\right)$. 
Projective bundles. Flat grundles appear in addressing the following question. Let $P \rightarrow X$ be a smooth fibre bundle with fibre $\mathbf{P}^{n-1}$ and structure group $\mathrm{PGL}_{n}(\mathbf{C})$. When does there exist a complex vector bundle $E \rightarrow X$ of rank $n$ with $\mathbf{P}(E) \cong P$ ? This is equivalent to asking for a complex line bundle $\lambda \rightarrow P$ whose restriction to the fibres is $\mathcal{O}(-1)$, for then $E$ is obtained from $\lambda$ by blowing down the zero-section.

To answer the question, we first reduce the structure group, by general principles, to $\mathrm{PU}_{n}$. Consider the short exact sequence

$$
0 \rightarrow S^{1} \rightarrow U_{n} \stackrel{\pi}{\longrightarrow} P U_{n} \rightarrow 1 .
$$

Let $\mathcal{U}=\left\{U_{i}\right\}_{i}$ be an acyclic covering of $X$ with trivializations $\left.P\right|_{U_{i}} \rightarrow$ $U_{i} \times \mathbf{P}^{n-1}$ and transition functions $G_{i j}: U_{i j} \rightarrow \mathrm{PU}_{n}$. Choose liftings $g_{i j}: U_{i j} \rightarrow \mathrm{U}_{n}$ with $\pi \circ g_{i j}=G_{i j}$ and define a 2-grundle over $\mathcal{U}$ by setting $\phi=0, A_{i}=0, A_{i j}=\frac{1}{n} d \log \operatorname{det} g_{i j}$ and $g_{i l k} I=g_{i j} g_{j k} g_{k i}$. Note that $\pi\left(g_{i j} g_{j k} g_{k i}\right) \equiv 1$ and so $g_{i j k} \in S^{1}$ and that $\left(A_{i}, A_{i j}, g\right)$ satisfy the grundle conditions of Section 5 . The class of the cocycle $g_{i j k}$ in $H_{\text {tor }}^{2}\left(X ; \mathcal{E}_{S^{1}}\right) \cong H_{\text {tor }}^{3}(X ; \mathbf{Z})$ represents the obstruction to the existence of the desired vector bundle $E$. This group $H_{\text {tor }}^{3}(X ; \mathbf{Z})$, called the topological Brauer group, was introduced by Grothendieck and Serre [9].

Hodge sparks. On a Riemannian manifold, the Greens operator $G$ provides an important source of sparks (see [17, Section 12] for more detials). Given an integrally flat $(k+1)$ cycle $R$ (such as a current chain without boundary), the Hodge spark of $R$ is defined by $\sigma(R)=-d^{*} G(R)$. It satisfies the spark equation

$$
d \sigma(R)=H(R)-R
$$

where $H(R)$ is the harmonic form determined by the class of $R$.

This is an example where the de Rham-Federer point of view is clearly superior to the other points of view. The holonomy map is easily computed for any cycle $S$ which does not meet $R$ by the formula

$$
h_{R}(S) \equiv \int_{S} \sigma(R) \quad(\bmod \mathbf{Z})
$$

If $R$ is a boundary, then $H(R)=0$ and the spark class of $\sigma(R)$ lies in the torus $H^{k}(X ; \mathbf{R}) / H^{k}(X ; \mathbf{Z})=\operatorname{ker} \delta_{1} \cap \operatorname{ker} \delta_{2}$. This "Jacobian" torus can be realized either as $\operatorname{Jac}^{k}=\operatorname{Har}^{k}(X) / \operatorname{Har}_{0}^{k}(X)$, harmonic forms modulo those with integral periods, or by duality as $\operatorname{Hom}\left(\operatorname{Har}_{0}^{k}(X), \mathbf{R} / \mathbf{Z}\right)$. The induced "Abel-Jacobi"map $j: B^{k+1} \rightarrow J a c^{k}$ on the space of boundaries can be realized directly by sending the boundary $R=d \Gamma$ to the spark class 
of $H(\Gamma)$, the harmonic form corresponding to $\Gamma$. That is $\sigma(R)$ and $H(R)$ are equivalent sparks.

We define boundaries in the kernel of the Abel-Jacobi map $j$ to be linearly equivalent to zero. These are the "principal boundaries" whose associated spark class is 0 . One has that a boundary $R=d \Gamma$ is linearly equivalent to zero iff

$$
\sigma(R)=\int_{\Gamma} \theta \quad(\bmod \mathbf{Z})
$$

for all harmonic $(n-k)$-forms $\theta$ with integral periods.

In summary, this example is best described from the de Rham-Federer point of view. See $[20,4]$ for a gerbe discussion in special cases.

Morse sparks. Suppose $\varphi_{t}$ is a Morse-Stokes flow [14] or more generally a finite-volume flow (see [14] and [23]). Then, for each critical point $p$, both the stable manifold $S_{p}$ and the unstable manifold $U_{p}$ have finite volume and define currents (by integration) also denoted by $S_{p}$ and $U_{p}$. Under the flow $\varphi_{t}$, each smooth form $\alpha$ has the limit

$$
P(\alpha)=\lim _{t \rightarrow \infty} \varphi_{t}^{*}(\alpha)=\sum_{p \in \mathrm{Cr}}\left(\int_{U_{p}} \alpha\right) S_{p}
$$

in the space of currents. For a form $\alpha$ whose residues $\int_{U_{p}} \alpha$ are integers, the limit $P(\alpha)=R$ is a rectifiable current, and $\alpha$ can be considered a "Thom form" for $\alpha$.

There exists a continuous linear operator $T$ from forms to currents which lowers degree by one. The Morse spark $T(\alpha)$ satisfies the spark equation

$$
d T(\alpha)=\alpha-R .
$$

The operator $T$ is induced by the kernel current $\mathbf{T} \equiv\left\{\left(x, \varphi_{t}(x)\right) \in X \times X\right.$ : $t \geq 0\}$. There is an abundance of interesting sparks coming from specific flows and forms. This is a case where the de Rham-Federer viewpoint seems the only way to construct the spark classes.

Curvature-driven sparks. When $H^{k}\left(X, S^{1}\right)=0$, the curvature form uniquely determines each spark class since $\delta_{1}: \widehat{\mathbf{H}}^{k}(X) \rightarrow \mathcal{Z}_{0}^{k+1}(X)$ is an isomorphism. By Poincaré duality, $H^{k}\left(X, S^{1}\right)$ vanishes if and only if $H_{k}(X, \mathbf{Z})$ vanishes. (Also note that $H^{k}\left(X, S^{1}\right)=0$ if and only if $H^{k}(X, \mathbf{R})=0$ and $H_{\text {tor }}^{k+1}(X, \mathbf{Z})=0$.) In this case, the holonomy point of view is transparent. Since each cycle $\Sigma$ of dimension $k$ is a boundary $\Sigma=d \Gamma$, the holonomy $h_{\phi}(\Sigma) \equiv \int_{\Gamma} \phi(\bmod \mathbf{Z})$ is well defined. $\left(\int_{\Gamma} \phi\right.$ is well defined modulo the periods of $\phi$.) 
Example: The Wess-Zumino term. Suppose $G$ is a compact simple simply-connected Lie group. Let $\Phi(X, Y, Z)=B(X,[Y, Z])$ denote the Car$\tan 3$-form (bi-invariant and d-closed) on $G$ where $B$ denotes the Killing form on the Lie algebra $\mathfrak{g}$. Since $H^{3}(G ; \mathbf{Z}) \cong \mathbf{Z}, \Phi$ may be normalized to be a generator. Also, $H^{2}(G ; \mathbf{Z})=0$, and hence $\Phi$ uniquely determines a class $w z \in \widehat{\mathbf{H}}^{2}(G)$.

As noted above, the holonomy map (as well as the differential character) point of view is transparent in this case. However, a de Rham-Federer spark also provides some geometric insight. Let $E: \mathfrak{g} \rightarrow G$ denote the exponential map. There exists a unique bounded star-shaped neighborhood $U$ of the origin in $\mathfrak{g}$ which is diffeomorphic, under $E$, to the open set $G-C$ where $C$ is the cut locus. The cut locus is a codimension-3 stratified set which naturally determines a current of degree 3 on $G$. If $a \in \mathcal{E}^{2}(\mathfrak{g})$ is chosen to satisfy the equation $d a=E^{*}(\Phi)$ on $\mathfrak{g}$, then $A=E_{*}\left(\chi_{U} a\right)$ is an $L_{\text {loc }}^{1}$-spark on $G$ with curvature form $\Phi$. In fact, one can show that on $G$

$$
d A=\Phi-[C] .
$$

Note that if $\bar{A}$ is another current on $G$ satisfying the equation (11.1), then $\bar{A}=A+d B$ for some $B \in \mathcal{D}^{\prime 1}(G)$ because $H^{1}(G)=0$. In particular $a$, and therefore also $A$, can be chosen to be $\mathrm{Ad}_{G}$-invariant. $\operatorname{Ad}_{G}$-invariant sparks satisfying (11.1) will be called Wess-Zumino sparks.

An even more explicit construction of an $\left(\mathrm{Ad}_{G}\right.$-invariant) Wess-Zumino spark is given by the taking the Hodge spark $A=-d^{*} G([C])$ of the cut locus $C$.

Torsion classes. Suppose $c \in \widehat{\mathbf{H}}^{k}(X)$ is a torsion class of torsion degree $n$. Then, since $n c=0$, the curvature satisfies $n \delta_{1}(c)=n \phi=0$. Hence, $c \in \operatorname{ker} \delta_{1} \equiv \widehat{\mathbf{H}}_{\text {flat }}^{k}(X)=H^{k}\left(X, S^{1}\right)$ is a flat class. Such classes $c \in H^{k}\left(X, S^{1}\right)$ can be related to elements in $H^{k}\left(X, \mathbf{Z}_{n}\right)$ as follows. The exact triple $0 \rightarrow$ $\mathbf{Z}_{n} \stackrel{1 / n}{\longrightarrow} S^{1} \stackrel{n}{\longrightarrow} S^{1} \rightarrow 0$ induces a long exact sequence $\cdots \rightarrow H^{k}\left(X, \mathbf{Z}_{n}\right) \stackrel{1 / n}{\longrightarrow}$ $H^{k}\left(X, S^{1}\right) \stackrel{n}{\longrightarrow} H^{k}\left(X, S^{1}\right) \longrightarrow \ldots$ Since $n c=0$, there exists an element $u \in H^{k}\left(X, \mathbf{Z}_{n}\right)$ whose image in $H^{k}\left(X, S^{1}\right)$ is $c$. Let $\beta$ denote the Bockstein map induced by $0 \rightarrow \mathbf{Z} \stackrel{n}{\longrightarrow} \mathbf{Z} \rightarrow \mathbf{Z}_{n} \rightarrow 0$. Then, the "divisor class" of $c$, namely $\delta_{2}(c) \in H^{k+1}(X, \mathbf{Z})$, can be computed more directly in terms of $u$. That is, $\beta(u)=\delta_{2}(c)$ in $H^{k+1}(X, \mathbf{Z})$. This fact follows from the commutativity of the following diagram.

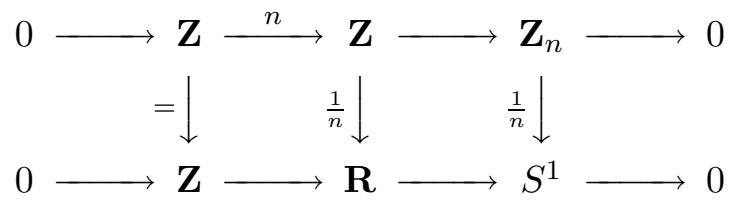


Since $H_{n \text {-torsion }}^{k}\left(X, S^{1}\right)=\operatorname{Hom}\left(H_{k}(X, \mathbf{Z}), \mathbf{Z}_{n}\right)$, the $n$-torsion elements in $\widehat{\mathbf{H}}^{k}(X)$ are given from the holonomy map point of view by

$$
\operatorname{Hol}_{n \text {-torsion }}(X) \cong \operatorname{Hom}\left(H_{k}(X, \mathbf{Z}), \mathbf{Z}_{n}\right) .
$$

The refined integer Stiefel-Whitney class. Suppose $F$ is an oriented rank $n$ real vector bundle. Then, the $k$ th Steifel-Whitney class of $F$ is a class $w_{k}(F) \in H^{k}\left(X ; \mathbf{Z}_{2}\right)$. Suppose $k<n$ is even (otherwise twist by the orientation bundle of $F)$. The image of $w_{k}(F)$ under the Bockstein map associated with $0 \rightarrow \mathbf{Z} \stackrel{2}{\longrightarrow} \mathbf{Z} \rightarrow \mathbf{Z}_{2} \rightarrow 0$ is the $(k+1)$ st integer Stiefel-Whitney class $W_{k+1}(F) \in H_{2 \text {-torsion }}^{k+1}(X ; \mathbf{Z})$. This defines a unique Stiefel-Whitney secondary class $\widehat{W}_{k+1}(F) \in H^{k}\left(X ; S^{1}\right) \subset \widehat{\mathbf{H}}^{k}(X)$, namely $\widehat{W}_{k+1}(F)$ is the image of $w_{k}(F)$ under the mapping $H^{k}\left(X ; \mathbf{Z}_{2}\right) \rightarrow H^{k}\left(X ; S^{1}\right)$ as in the paragraph above.

Linear dependency currents and Stiefel-Whitney sparks. Suppose $F$ is a rank $n$ real vector bundle. Following [18], suppose $\alpha=\left(\alpha_{1}, \ldots, \alpha_{n-k}\right)$ is an atomic collection of sections of $F$. Suppose $F$ is oriented (or otherwise twist by the orientation bundle of $F$ ). Assume $k$ is even. Then, there exists a $d$-closed locally integrally flat current $L D(\alpha)$ of degree $k+1$, whose support is contained in the linear dependency set of the collection of sections. Furthermore, if $\beta$ is another atomic collection of sections, then

$$
L D(\alpha)-L D(\beta)=d R
$$

where $R$ is a locally rectifiable current.

It is proven in [18] that the class of $L D(\alpha)$ in $H^{k+1}(X ; \mathbf{Z})$ is $W_{k+1}(F)$, the $(k+1)$ st integer Stiefel-Whitney class of $F$.

Now, given a metric connection on $F$, Zweck [25] constructs a canonical spark $S_{k+1}(\alpha)$ which is a current with $L_{\text {loc }}^{1}$-coefficients satisfying the (flat) spark equation

$$
d S_{k+1}(\alpha)=-L D(\alpha)
$$

on $X$. Furthermore, Zweck [25] proves a spark comparison formula

$$
S_{k+1}(\alpha)-S_{k+1}\left(\alpha^{\prime}\right)=T+d L
$$

with $T$ locally integrally flat and $L$ an $L_{\text {loc }}^{1}$-current. That is, there is a well defined class

$$
\left[S_{k+1}(\alpha)\right] \in H^{k}\left(X ; S^{1}\right) \subset \widehat{\mathbf{H}}^{k}(X)
$$

Zweck [25] proves that this spark class, which refines $W_{k+1}(F)$ is the image of the $k$ th Stiefel-Whitney class $w_{k}(F) \in H^{k}\left(X ; \mathbf{Z}_{2}\right)$ under the map $(1 / 2)$ : $H^{k}\left(X ; \mathbf{Z}_{2}\right) \rightarrow H^{k}\left(X ; S^{1}\right)$. This proves that the spark class of $S_{k+1}(\alpha)$ is the refined integer $(k+1)$ st Stiefel-Whitney class $\widehat{W}_{k+1}(F)$, discussed above. 


\section{References.}

[1] J.-L. Brylinski, Loop Spaces, Characteristic Classes and Geometric Quantization, Birkhauser, Boston, 1993.

[2] J. Cheeger, Multiplication of Differential Characters, Instituto Nazionale di Alta Mathematica, Symposia Mathematica XI, (1973), 441-445.

[3] J. Cheeger and J. Simons, Differential Characters and Geometric Invariants, in Geometry and Topology, Lect. Notes in Math. no. 1167, Springer-Verlag, New York, 1985, 50-80.

[4] D. S. Chatterjee, On the construction of abelian gerbs, Ph. D. Thesis, Cambridge, 1998.

[5] G. de Rham, Variétés Différentiables, formes, courants, formes harmoniques, Hermann, Paris, 1955.

[6] H. Federer, Geometric Measure Theory, Springer-Verlag, New York, 1969.

[7] D. Freed and E. Witten, Anomalies in string theory with D-branes, Asian J. Math., 3, (1999), 819-851.

[8] H. Gillet and C. Soulé, Arithmetic chow groups and differential characters, in Algebraic K-theory: Connections with Geometry and Topology (Jardine and Snaith, eds.), Kluwer Academic Publishers, 1989, 30-68.

[9] A. Grothendieck, Le groupe de Brauer, Dix Exposés sur la Cohomology des Schémas, North Holland Publ. Co, 1968.

[10] B. Harris, Differential characters and the Abel-Jacobi map, in Algebraic K-theory: Connections with Geometry and Topology, (Jardine and Snaith, eds), Kluwer Academic Publishers, 1989, 69-86.

[11] F.R. Harvey and H.B. Lawson, Jr., A theory of characteristic currents associated with a singular connection, Astérisque, 213 Société Math. de France, Paris, 1993.

[12] F.R. Harvey and H.B. Lawson, Jr., Geometric residue theorems, Amer. J. Math., 117, (1995), 829-873. 
[13] F.R. Harvey and H.B. Lawson, Jr., Lefschetz-Pontrjagin duality for differential characters, Anais da Academia Brasileira de Ciências, 73, (2001), 145-159. ArXiv:math.DG/0512528

[14] F.R. Harvey and H.B. Lawson, Jr., Finite volume flows and Morse theory, Ann. Math., 153, (2001), 1-25. ArXiv:math.DG/0101268

[15] F.R. Harvey and H.B. Lawson, Jr., D-bar sparks, I, Stony Brook Preprint (2003). ArXiv:math.DG/0512247

[16] F.R. Harvey and H.B. Lawson, Jr., D-bar sparks, II - Arithmetic sparks, (to appear).

[17] F.R. Harvey, H.B. Lawson, Jr. and John Zweck, The deRham-Federer theory of differential characters and character duality, Amer. J. Math., 125, (2003), 791-847. ArXiv:math.DG/0512251

[18] F.R. Harvey and John Zweck, Stiefel-Whitney currents, J. Geom. Analysis, 8, (1998), 809-844.

[19] F.R. Harvey and John Zweck, Divisors and Euler sparks of atomic sections, Indiana Univ. Math. Jour., 50, (2001), 243-298.

[20] N. Hitchin, Lectures on special lagrangian submanifolds, in Winter School on Mirror Symmetry, Vector Bundles and Lagrangian Submanifolds (Cambridge, MA, 1999), AMS/IP Stud. Adv. Math., 23, Amer. Math. Soc., Providence, RI, (2001), 151-182. ArXiv:math.DG/9907034 (1999).

[21] R. Picken, A cohomological description of Abelian bundles and gerbes, Preprint of CEMAT, Lisboa, Portugal, math.DG/0305147 9 May 03.

[22] M. Mackaay and R. Picken, Holonomy and parallel transport for Abelian gerbes, Adv. Math., 170, (2002), 287-339.

[23] G. Minervini, A current approach to Morse and Novikov theories, Ph.D. thesis, Universita "La Sapienza", Roma 2002.

[24] J. Simons, Characteristic forms and transgression: characters associated to a connection, Stony Brook preprint, (1974).

[25] John Zweck, Stiefel-Whitney sparks, Houston Journal of Math., 27(2), (2001), 325-351. 